\title{
The everchanging pulsating white dwarf GD358 ^
}

S. O. Kepler ${ }^{1}$, R. E. Nather ${ }^{2}$, D. E. Winget ${ }^{2}$, A. Nitta ${ }^{3}$, S. J. Kleinman ${ }^{3}$, T. Metcalfe ${ }^{2,4}$, K. Sekiguchi $^{5}$, Jiang Xiaojun ${ }^{6}$, D. Sullivan ${ }^{7}$, T. Sullivan ${ }^{7}$, R. Janulis ${ }^{8}$, E. Meistas ${ }^{8}$, R. Kalytis ${ }^{8}$, J. Krzesinski ${ }^{9}$, W. Ogłoza ${ }^{9}$, S. Zola ${ }^{10}$, D. O'Donoghue ${ }^{11}$, E. Romero-Colmenero ${ }^{11}$, P. Martinez ${ }^{11}$, S. Dreizler ${ }^{12}$, J. Deetjen ${ }^{12}$, T. Nagel ${ }^{12}$, S. L. Schuh ${ }^{12}$, G. Vauclair ${ }^{13}$, Fu Jian Ning ${ }^{13}$, M. Chevreton ${ }^{14}$, J.-E. Solheim ${ }^{15}$, J. M. Gonzalez Perez ${ }^{15}$, F. Johannessen ${ }^{15}$, A. Kanaan ${ }^{16}$, J. E. Costa ${ }^{1}$, A. F. Murillo Costa ${ }^{1}$, M. A. Wood ${ }^{17}$, N. Silvestri ${ }^{17}$, T. J. Ahrens ${ }^{17}$, A. K. Jones ${ }^{18, \star \star}$, A. E. Collins ${ }^{19, \star \star}$, M. Boyer ${ }^{20, \star \star}$, J. S. Shaw ${ }^{21}$, A. Mukadam ${ }^{2}$, E. W. Klumpe ${ }^{22}$, J. Larrison ${ }^{22}$, S. Kawaler ${ }^{23}$, R. Riddle ${ }^{23}$, A. Ulla ${ }^{24}$, and P. Bradley ${ }^{25}$

1 Instituto de Física da UFRGS, Porto Alegre, RS - Brazil

e-mail: kepler@if.ufrgs.br

2 Department of Astronomy \& McDonald Observatory, University of Texas, Austin, TX 78712, USA

3 Sloan Digital Sky Survey, Apache Pt. Observatory, PO Box 59, Sunspot, NM 88349, USA

${ }^{4}$ Harvard-Smithsonian Center for Astrophysics, 60 Garden Street, Cambridge, MA 02138, USA e-mail: travis@whitedwarf.org

5 Subaru National Astronomical Observatory of Japan e-mail: kaz@subaru.naoj.org

${ }^{6}$ Beijing Astronomical Observatory, Academy of Sciences, Beijing 100080, PR China e-mail: jiang@astro.as .utexas . edu

7 University of Victoria, Wellington, New Zealand

${ }^{8}$ Institute of Theoretical Physics and Astronomy, Gostauto 12, Vilnius 2600, Lithuania

9 Mt. Suhora Observatory, Cracow Pedagogical University, Ul. Podchorazych 2, 30-084 Cracow, Poland

10 Jagiellonian University, Krakow, Poland e-mail: zola@oa.uj.edu.pl

11 South African Astronomical Observatory

12 Universitat Tübingen, Germany

13 Université Paul Sabatier, Observatoire Midi-Pyrénées, CNRS/UMR5572, 14 av. E. Belin, 31400 Toulouse, France

14 Observatoire de Paris-Meudon, DAEC, 92195 Meudon, France e-mail: chevreton@obspm.fr

15 Institutt for fysikk, 9037 Tromso, Norway

16 Departamento de Física, Universidade Federal de Santa Catarina, CP 476, CEP 88040-900, Florianópolis, Brazil e-mail: kanaan@fsc.ufsc.br

17 Dept. of Physics and Space Sciences \& The SARA ${ }^{\star}$ Observatory, Florida Institute of Technology, Melbourne, FL 32901, USA

18 University of Florida, 202 Nuclear Sciences Center Gainesville, FL 32611-8300, USA

19 Johnson Space Center, 2101 NASA Road 1, Mail Code GT2, Houston, TX 77058, USA

${ }^{20}$ University of Minnesota, Department of Physics \& Astronomy, 116 Church St. S.E., Minneapolis, MN 55455, USA

${ }^{21}$ University of Georgia at Athens, Department of Physics and Astronomy, Athens, GA 30602-2451, USA

${ }^{22}$ Middle Tennessee State University, Department of Physics and Astronomy Murfreesboro, TN 37132, USA

23 Department of Physics and Astronomy, Iowa State University, Ames, IA 50011, USA

24 Universidade de Vigo, Depto. de Fisica Aplicada, Facultade de Ciencias, Campus Marcosende-Lagoas, 36200 Vigo (Pontevedra), Spain e-mail: ulla@uvigo.es

25 Los Alamos National Laboratory, X-2, MS T-085 Los Alamos, NM 87545, USA

Received 6 December 2002 / Accepted 21 January 2003

Send offprint requests to: S. O. Kepler, e-mail: kepler@if.ufrgs.br

* Tables 1 to 4 and 11 and Figs. 4, 5, 6, 10, 11 and 12 are available only in the electronic form at http://www . edpsciences.org

$\star \star$ Southeastern Association for Research in Astronomy (SARA) NSF-REU Student. 
Abstract. We report 323 hours of nearly uninterrupted time series photometric observations of the DBV star GD 358 acquired with the Whole Earth Telescope (WET) during May 23rd to June 8th, 2000. We acquired more than 232000 independent measurements. We also report on 48 hours of time-series photometric observations in Aug 1996. We detected the non-radial $g$-modes consistent with degree $\ell=1$ and radial order 8 to 20 and their linear combinations up to 6th order. We also detect, for the first time, a high amplitude $\ell=2$ mode, with a period of $796 \mathrm{~s}$. In the 2000 WET data, the largest amplitude modes are similar to those detected with the WET observations of 1990 and 1994 , but the highest combination order previously detected was 4th order. At one point during the 1996 observations, most of the pulsation energy was transferred into the radial order $k=8$ mode, which displayed a sinusoidal pulse shape in spite of the large amplitude. The multiplet structure of the individual modes changes from year to year, and during the 2000 observations only the $k=9$ mode displays clear normal triplet structure. Even though the pulsation amplitudes change on timescales of days and years, the eigenfrequencies remain essentially the same, showing the stellar structure is not changing on any dynamical timescale.

Key words. stars: white dwarfs - stars: variables: general - stars: oscillations - stars: individual: GD 358 - stars: evolution

\section{Introduction}

GD 358, also called V777 Herculis, is the prototype of the DBV class of white dwarf pulsators. It was the first pulsating star detected based on a theoretical prediction (Winget et al. 1985), and is the pulsating star with the largest number of periodicities detected after the Sun. Detecting as many modes as possible is important, as each periodicity detected yields an independent constraint on the star's structure. The study of pulsating white dwarf stars has allowed us to measure the stellar mass and composition layers, to probe the physics at high densities, including crystallization, and has provided a chronometer to measure the age of the oldest stars and consequently, the age of the Galaxy.

Robinson et al. (1982) and Kepler (1984) demonstrated that the variable white dwarf stars pulsate in non-radial gravity modes. Beauchamp et al. (1999) studied the spectra of the pulsating DBs to determine their instability strip at $22400 \leq$ $T_{\text {eff }} \leq 27800 \mathrm{~K}$, and found $T_{\text {eff }}=24900 \mathrm{~K}, \log g=7.91$ for the brightest DBV, GD $358(V=13.85)$, assuming no photospheric H, as confirmed by Provencal et al. (2000). Provencal et al. studied the HST and EUVE spectra, deriving $T_{\text {eff }}=$ $27000 \pm 1000 \mathrm{~K}$, finding traces of carbon in the atmosphere $[\log (\mathrm{C} / \mathrm{He})=-5.9 \pm 0.3]$ and a broadening corresponding to $v \sin i=60 \pm 6 \mathrm{~km} \mathrm{~s}^{-1}$. They also detected Ly $\alpha$ that is probably interstellar. Althaus \& Benvenuto (1997) demonstrated that the Canuto et al. (1996, hereafter CGM) self consistent theory of turbulent convection is consistent with the $T_{\text {eff }} \simeq 27000 \mathrm{~K}$ determination, as GD 358 defines the blue edge of the DBV instability strip. Shipman et al. (2002) extended the blue edge of the DBV instability strip by finding that the even hotter star PG0112+104 is a pulsator.

Winget et al. (1994) reported on the analysis of 154 hours of nearly continuous time series photometry on GD 358, obtained during the Whole Earth Telescope (WET) run of May 1990. The Fourier temporal spectrum of the light curve is dominated by periodicities in the range $1000-2400 \mu \mathrm{Hz}$, with more than 180 significant peaks. They identify all of the triplet frequencies as having degree $\ell=1$ and, from the details of their triplet $(k)$ spacings, from which Bradley \& Winget (1994) derived the total stellar mass as $0.61 \pm 0.03 M_{\odot}$, the mass of the outer helium envelope as $2.0 \pm 1.0 \times 10^{-6} M_{*}$, the luminosity as $0.050 \pm 0.012 L_{\odot}$ and, deriving a temperature and bolometric correction, the distance as $42 \pm 3 \mathrm{pc}$. Winget et al. (1994) found changes in the $m$ spacings among the triplet modes, and by assuming the rotational splitting coefficient $C_{\ell, k}(r)$ depends only on radial overtone $k$ and the rotation angular velocity $\Omega(r)$, interpret the observed spacing as strong evidence of radial differential rotation, with the outer envelope rotating some 1.8 times faster than the core. However, Kawaler et al. (1999) find that the core rotates faster than the envelope when they perform rotational splitting inversions of the observational data. The apparently contradictory result is due to the presence of mode trapping and the behavior of the rotational splitting kernel in the core of the model. Winget et al. also found significant power at the sums and differences of the dominant frequencies, indicating that non-linear processes are significant, but with a richness and complexity that rules out resonant mode coupling as a major cause.

We show that in the WET data set reported here (acquired in 2000), only 12 of the periodicities can be identified as independent $g$-mode pulsations, probably all different radial overtones $(k)$ with same spherical degree $\ell=1$, plus the azimuthal $m$ components for $k=8$ and 9 . The high amplitude with a period of $796 \mathrm{~s}$ is identified as an $\ell=2$ mode; it was not present in the previous data sets. Most, if not all, of the remaining periodicities are linear combination peaks of these pulsations. Considering there are many more observed combination frequencies than available eigenmodes, we interpret the linear combination peaks as caused by non-linear effects, not real pulsations. This interpretation is consistent with the proposal by Brickhill (1992) and $\mathrm{Wu}$ (2001) that the combination frequencies appear by the non-linear response of the medium. Recently, van Kerkwijk et al. (2000) and Clemens et al. (2000) show that most linear combination peaks for the DAV G29-38 do not show any velocity variations, while the eigenmodes do. However, Thompson et al. (2003) argue that some combination peaks do show velocity variations.

As a clear demonstration of the power of asteroseismology, Metcalfe et al. (2001) and Metcalfe et al. (2002) used GD 358 observed periods from Winget et al. (1994) and a genetic algorithm to search for the optimum theoretical model with static diffusion envelopes, and constrained the ${ }^{12} \mathrm{C}(\alpha, \gamma){ }^{16} \mathrm{O}$ cross section, a crucial parameter for many fields in astrophysics and difficult to constrain in terrestrial laboratories. Montgomery et al. (2001) also used the observed pulsations to constrain the diffusion of ${ }^{3} \mathrm{He}$ in white dwarf stars. They show their best model for GD 358 has $\mathrm{O} / \mathrm{C} /{ }^{4} \mathrm{He} /{ }^{3} \mathrm{He}$ structure, $T_{\text {eff }}=22300 \pm 500 \mathrm{~K}, M_{*}=0.630 \pm 0.015 M_{\odot}$, a thick He layer, $\log M\left({ }^{4} \mathrm{He}\right) / M_{*}=(-2.79 \pm 0.06)$, distinct from the thin layer, $\log M\left({ }^{4} \mathrm{He}\right) / M_{*}=\left(-5.70 \pm_{-0.30}^{+0.18}\right)$, proposed by 
Bradley \& Winget (1994). Montgomery, Metcalfe, \& Winget's model had $\log M\left({ }^{3} \mathrm{He}\right) / M_{*}=(-7.49 \pm 0.12)$, but Wolff et al. (2002) did not detect any ${ }^{3} \mathrm{He}$ in the spectra of all the DBs they observed. On the other hand, Dehner \& Kawaler (1995), Brassard \& Fontaine (2002), and Fontaine \& Brassard (2002) show that a thin helium envelope is consistent with the evolutionary models starting at PG1159 models and ending as DQs, as diffusion is still ongoing around $25000 \mathrm{~K}$ and lower temperatures. Therefore there could be two transition zones in the envelope, one between the $\mathrm{He}$ envelope and the $\mathrm{He} / \mathrm{C} / \mathrm{O}$ layer, where diffusion is still separating the elements, and another transition between this layer and the $\mathrm{C} / \mathrm{O}$ core.

Gautschy \& Althaus (2002) calculated nonadiabatic pulsation properties of DB pulsators using evolutionary models including the CGM full-spectrum turbulence theory of convection and time-dependent element diffusion. They show that up to 45 dipole modes should be excited, with periods between $335 \mathrm{~s}$ and $2600 \mathrm{~s}$ depending on the mass of the star, though their models did not include pulsation-convection coupling. They obtain a trapping-cycle length of $\Delta k=5 \rightarrow 7$, and the quadrupole modes showed instabilities comparable to the dipole modes.

Buchler et al. (1997) show that if there is a resonance between pulsation modes, even if the mode is stable, its amplitude will be necessarily nonzero. They also point out that in case of amplitude saturation, it is the smaller adjacent modes that show the largest amplitude variation, not the main modes. However, if the combination peaks are not real modes in a physical sense, just non-linear distortion by the medium, it is not clear that one would have resonant (mode-coupling) between the combination peaks and real modes.

When the Whole Earth Telescope observed GD 358 in 1990, 181 periodicities were detected, but only modes from radial order $k=8$ to 18 were identified, most of them showing triplets, consistent with the degree $\ell=1$ identification. In fact, the observed period spacing is consistent with the measured parallax only if the observed pulsations have degree $\ell=1$ (Bradley \& Winget 1994).

Vuille et al. (2000) studied the 342 hours of Whole Earth Telescope data obtained in 1994, showing again modes with $k=8$ to 18 , and discovered up to 4 th-order cross-frequencies in the power spectra. They compared the amplitudes and phases observed with those predicted by the pulsation-convection interaction proposed by Brickhill (1992), and found reasonable agreement.

Note that the number of nodes in the radial direction $k$ cannot be determined observationally and rely on a detailed comparison of the observed periods with those predicted by pulsation models.

\section{Observations}

We report here two data sets: $48 \mathrm{hr}$ of time series photometry acquired in August 1996, with the journal of observations presented in Table 1 . The second data set consists of 323 hours acquired in May-June 2000 (see Tables 2 and 3 for the observing $\log$ ). Both of these data sets were obtained simultaneously with time resolved spectroscopy with the Hubble Space Telescope, which will be reported elsewhere.

In 1996, the observations were obtained with three channel time series photometry using bi-alkali photocathodes (Kleinman et al. 1996), in Texas, China, and Poland. During 23 May to 23 June, 2000, we observed GD 358 mainly with two and three channel time series photometers using bi-alkali photocathodes and a time resolution of 5 s. The May-June 2000 run used 13 telescopes composing the Whole Earth Telescope. The telescopes, ranging from $60 \mathrm{~cm}$ to $256 \mathrm{~cm}$ in diameter, were located in Texas, Arizona, Hawaii, New Zealand, China, Lithuania, Poland, South Africa, France, Spain, Canary Islands, and Brazil. As the pulsations in white dwarf stars are in phase at different wavelengths (Robinson et al. 1982), we used no filters, to maximize the detected signal.

Each run was reduced and analyzed as described by Nather et al. (1990) and Kepler (1993), correcting for extinction through an estimated local coefficient, and sky variations measured continuously on three channel and CCD observations, or sampled frequently on two channel photometers. The second channel of the photometer monitored a nearby star to assure photometric conditions or correct for small non-photometric conditions. The CCD measurements were obtained with different cameras which are not described in detail here. At least two comparison stars were in each frame and allowed for differential weighted aperture photometry. The consecutive data points were 10 to $30 \mathrm{~s}$ apart, depending if the CCDs were frame transfer or not.

After this preliminary reduction, we brought the data to the same fractional amplitude scale and converted the middle of integration times to Barycentric Coordinated Time TCB (Standish 1998). We then computed a Discrete Fourier Transform (DFT) for the combined 2000 data, shown in Fig. 1. Due to poor weather conditions during the run, our coverage is not continuous, causing gaps in the observed light curve; these gaps produce aliases in the Fourier transform. At the bottom of Fig. 1 we present the spectral window, the Fourier transform of a single sinusoid sampled exactly as the real data. It shows the pattern of peaks each individual frequency in the data introduces in the DFT.

The Fourier spectra displayed in Fig. 1 looks similar to the ones obtained in 1990 and 1994 (see Fig. 2), but the amplitude of all the modes changed significantly. As we describe in more detail later, the most striking feature of the 2000 data is the absence of triplets, except for $k=9$. The 1996 data are even more unusual than the WET runs due to the observation of amplitude changes over an unprecedented short time. We describe these observations in more detail in Sect. 3. We then describe the 2000 observations and our interpretations of them in Sects. 4 through 7.

\section{Changes in the dominant mode in 1996}

We observed GD 358 in August 1996 to provide simultaneous observations to compare to HST time resolved spectroscopy made on August 16. The 1996 data covers 10 days of the most remarkable amplitude behavior ever seen in a pulsating white dwarf. Observing this behavior is serendipitous, as individual 

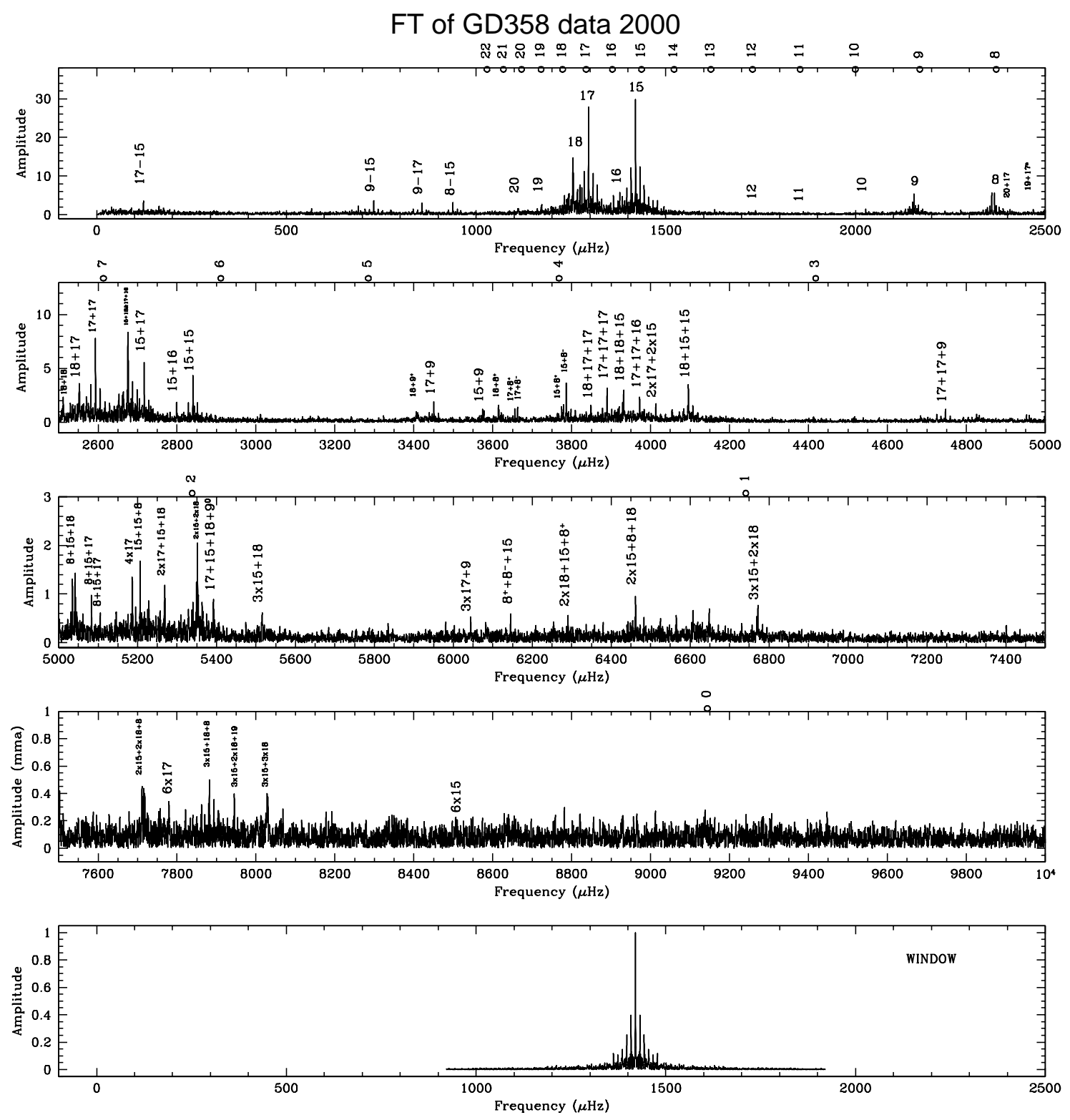

Fig. 1. Fourier transform of the 2000 data set. The main power is concentrated in the region between $1000 \mu \mathrm{Hz}$ and $2500 \mu \mathrm{Hz}$. The marks on top of the graph are the asymptotic equally spaced periods prediction, and the numbers represent the radial order $k$ value, with Winget et al. (1994) identification. The vertical scale on each panel are adjusted to accommodate the large range of amplitudes shown, and the noise level which decreases from $0.29 \mathrm{mma}$ up to $3000 \mu \mathrm{Hz}$ to $0.19 \mathrm{mma}$ upwards.

observers and the WET have observed GD 358 off and on for 20 years without seeing this sort of behavior.

Figure 3 shows how the amplitude of the $k=8 P=$ 423 s mode changed with time during our observations in August 1996. The amplitude changes we saw in our optical data are unprecedented in the observations of pulsating white dwarf stars; no report has been made of such a large amplitude variation in such a short amount of time. Here we describe what we found in our data.

The lightcurves acquired in August 1996 are displayed in Fig. 4 and the Fourier transform for two lightcurves is shown in Fig. 5. Those in the first and second panels of Fig. 4 look very different from each other. The Fourier transform of the lightcurve from the first panel is similar to that from the 1994 WET data where we identified over 100 individual periodicities, while the Fourier transform of the second panel is dominated by only a single periodicity (Fig. 5); this represents a complete change in the mode structure, as well as the period of the dominant mode, in about one day!

In run an-0034, the $k=8 P=423$ s mode's amplitude is $170 \mathrm{mma}$, which is the largest amplitude we have ever seen for this mode. To check for additional pulsation power (perhaps lower amplitude pulsations dwarfed by the $423 \mathrm{~s}$ mode power), we prewhitened the an-0034 lightcurve by the $423 \mathrm{~s}$ mode. Prewhitening subtracts a sinusoid with a specified amplitude, phase and period from the original lightcurve, and it helps us look for smaller amplitude pulsations by eliminating the alias pattern of the dominant pulsation mode from the Fourier transform. In Fig. 6, we show the Fourier transform of the an-0034 lightcurve both before and after prewhitening. We see now that 
Fourier Transform of GD358 data
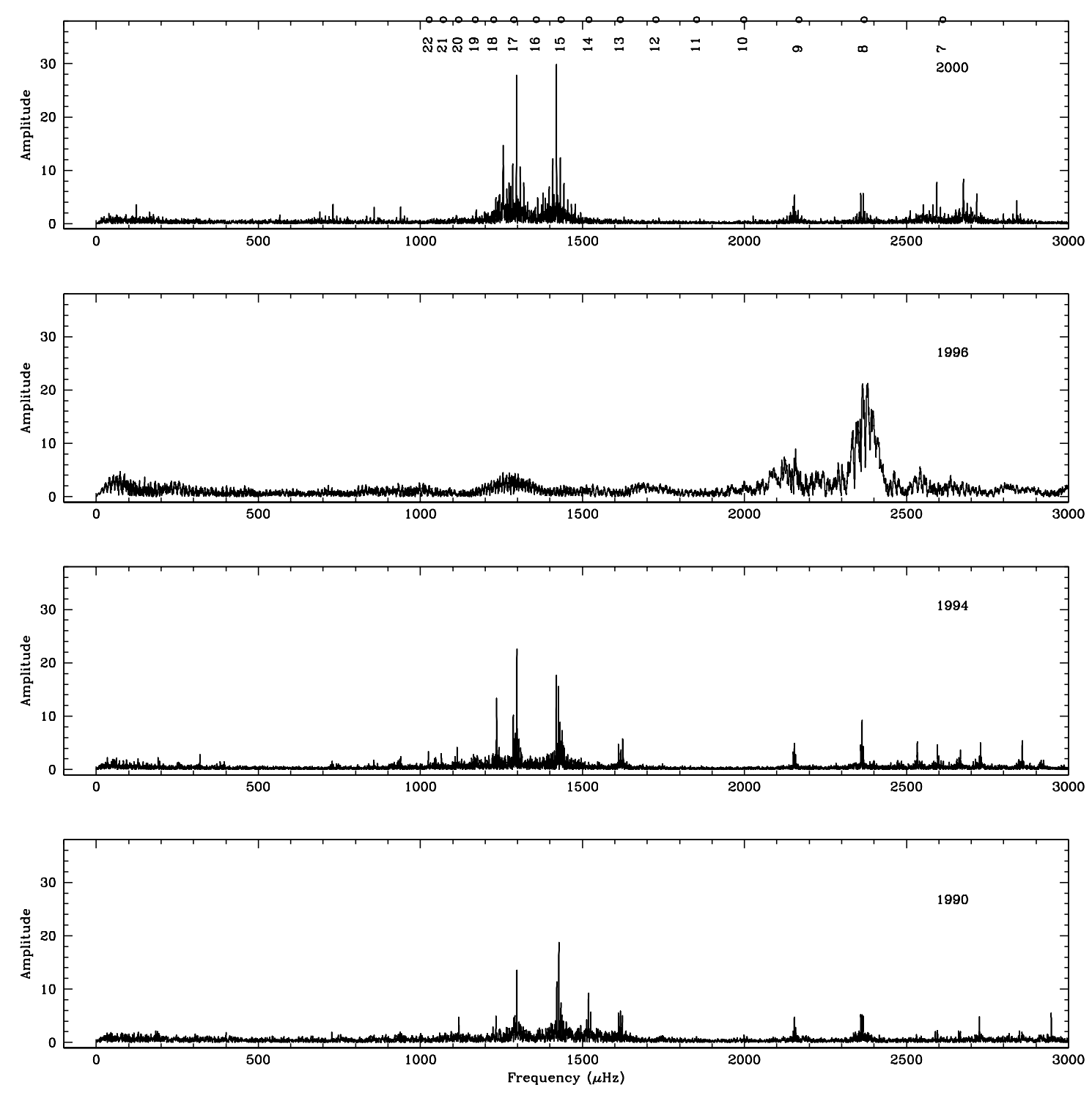

Fig. 2. Fourier transform of the GD 358 data, year by year.

GD 358 was indeed dominated entirely by a single mode at a different period from the dominant mode a day earlier. We refer to this event by the musical term "forte", or more informally as the "whoopsie".

Given the spectacular behavior of GD 358 in August 1996, we obtained follow-up observations in September 1996 and April 1997. Table 4 shows the journal of observations for the September 1996 and April 1997 data. The lightcurve and the power spectrum during these observations (Fig. 7) seem to have returned to the more or less normal state seen in the past (Fig. 2), not the unusually high amplitude state it was in in August 1996 (Fig. 5).

We show the lightcurves of GD 358 at three different times in Fig. 8. The middle panel shows the lightcurve when the amplitude of the $423 \mathrm{~s}$ mode was at its largest. The lightcurve looks almost sinusoidal, with the single $423 \mathrm{~s}$ mode in the power spectrum. The result of this is that we obtain similar values for the peak-to-peak semi-amplitudes and the
FT amplitude of the $423 \mathrm{~s}$ mode; this implies that a single spherical harmonic is a good representation of the stellar pulsation at this time. The other two lightcurves, however, each containing several pulsation modes, are less sinusoidal. If the non-sinusoidal nature of a lightcurve comes from the fact that many modes are present simultaneously, then one would expect the shape of the lightcurve to be sinusoidal only when it is pulsating in a single mode. On the other hand, in the August 1996 sinusoidal lightcurve, the peak-to-peak light variation was about $44 \%$ of the star's average light in the optical. We would expect such a large light variation to introduce nonlinear effects into the lightcurve, even if the star is pulsating in a single mode, causing the lightcurve to look nonsinusoidal. Thus, the nearly sinusoidal shape of our lightcurves (Fig. 8) is a mystery, except for the theoretical models of Ising \& Koester (2001), which predict sinusoidal shapes for large amplitude modes even with the nonlinear response of the envelope. 


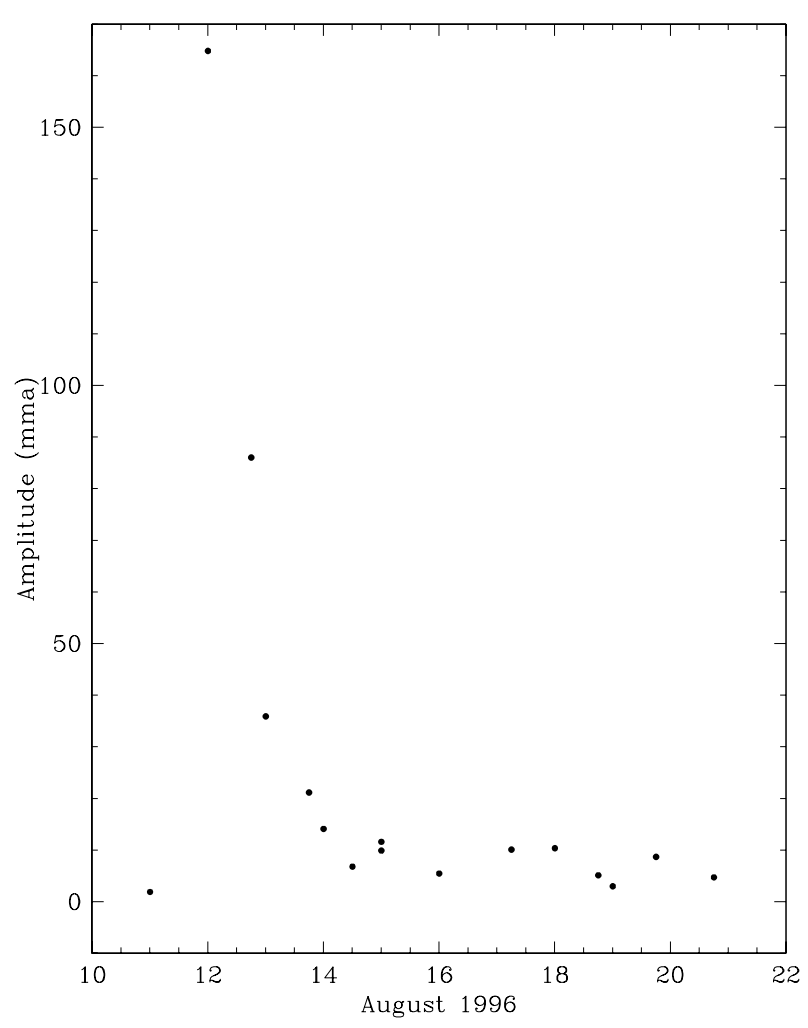

Fig. 3. The amplitude modulation of GD 358423 s mode observed in the optical in August, 1996. The timescale of this change is surprisingly short. We have never observed such a fast amplitude modulation in any of the pulsating white dwarf stars.

After the $P=423 \mathrm{~s}$ mode reached its highest amplitude in run an-0034, the $k=9 P=464 \mathrm{~s}$ mode started to grow and the $423 \mathrm{~s}$ became smaller, but there was still very little sign of the usually dominant $k=17 P=770$ s mode. In Fig. 2, we present the Fourier amplitude spectra of the light curves obtained each year, 2000 on top, 1996, 1994, and 1900 on the bottom, on the same vertical scale. Note that the 1996 data set is low resolution, because of its smaller amount of data. It is clear that the periodicities change amplitude from one data set to the other. It is important to notice that the periodicities, when present, have similar frequencies over the years. The amplitudes change, and even subcomponents (different $m$ values) may appear and disappear, but when they are present, they have basically the same frequencies (typically to within $1 \mu \mathrm{Hz}$ ).

In the September 1996 data, the Fourier transform shows that GD 358 is pulsating in periods similar to what we are familiar with from the WET data of 1990, although the highest peaks are at $1082 \mu \mathrm{Hz}, 2175 \mu \mathrm{Hz}$ and $2391 \mu \mathrm{Hz}$. The very limited data set and the complex pulsating structure of the star makes interpretation of these peaks difficult (Fig. 7). It is not until the data taken in April 1997 when we observe the $770 \mathrm{~s}$ mode as the highest amplitude mode in the Fourier transform, as in 1990 and 1994. We do not have data to fill in the gap between September 1996 and April 1997 to see how the amplitude changed, but even by August 19th, the modes at $k=15$ and 18 were already starting to appear. The time scale which the star took to change from its normal multi-mode state to a single mode pulsator was very short, about one day.
The reverse transition started one week after the event. An estimate of the total energy observed in pulsations is best obtained by measuring the peak-to-peak amplitudes in the light curves directly, instead of adding the total power from all the modes. For the largest amplitude run in 1996, an-0034, observed with the $2.1 \mathrm{~m}$ telescope at McDonald, we estimate a peak-to-peak semi-amplitude of $220 \mathrm{mma}$. For comparison, the measured Fourier amplitude for the $k=8$ mode for that run is 170 mma. For two runs at the same telescope in 2000, we obtain a peakto-peak semi-amplitude of $120 \mathrm{mma}$. Again for comparison, the Fourier amplitudes of the large modes present are $30 \mathrm{mma}$, but there are several modes, and many combination peaks. As the observed pulsation energy is quadratic in the amplitudes and the frequencies, it corresponds to an increase of around $34 \%$ in the radiated energy by pulsations, from the amplitudes, plus a factor of 2.8 from the frequency. Just two days after the "forte", the peak-to-peak amplitude decreased by a factor of 5, but during our observations a month later, it had already increased to its pre-"forte" value. It is important to notice that the observed amplitude is not directly a measurement of the physical amplitude, as there are several factors that typically depend on $\ell$, including: geometrical cancellation, inclination effects, kinetic energies associated with the oscillatory mass motions, together with a term that depends on the frequency of pulsation squared. If we assume that the inclination angle of the pulsation axis to our line of sight does not change, and that the $\ell$ values of the dominant modes do not change, then it must be the $\ell$ distribution of the combination frequencies that changes and produces a difference in the peak-to-peak variations in the light curve, if the total energy is conserved. This is plausible, as relatively small variations in the amplitudes of the dominant periods can dramatically change the amplitudes of the linear combination frequencies, but not necessary.

\section{Main periodicities in the 2000 WET data}

\subsection{Assumptions and ground rules used in this work}

We observed GD 358 as the primary target in May-June of 2000 to provide another "snapshot" of the behavior of GD 358 with minimal alias problems. For the period May 23rd to June 8th, this run provided coverage $(\sim 80 \%)$ that was intermediate between the 1994 run ( $86 \%$ coverage) and the 1990 run (with $69 \%$ coverage). The 2000 WET run had several objectives: 1) look for additional modes besides the known $\ell=1$ $k=8$ through 18 modes; 2) investigate the multiplet splitting structure of the pulsation modes; 3 ) look for amplitude changes of the known modes; 4) determine the structure of the "combination peaks", including the maximum order seen; and 5) provide simultaneous observations for HST time resolved spectroscopy.

Before we can start interpreting the peaks in the FT, we need to select an amplitude limit for what constitutes a "real" peak versus a "noise" peak. Kepler (1993) and SchwarzenbergCzerny (1991, 1999), following Scargle (1982), demonstrated that non-equally spaced data sets and multiperiodic light curves, as all the Whole Earth Telescope data sets are, do not have a normal noise distribution, because the residuals are 


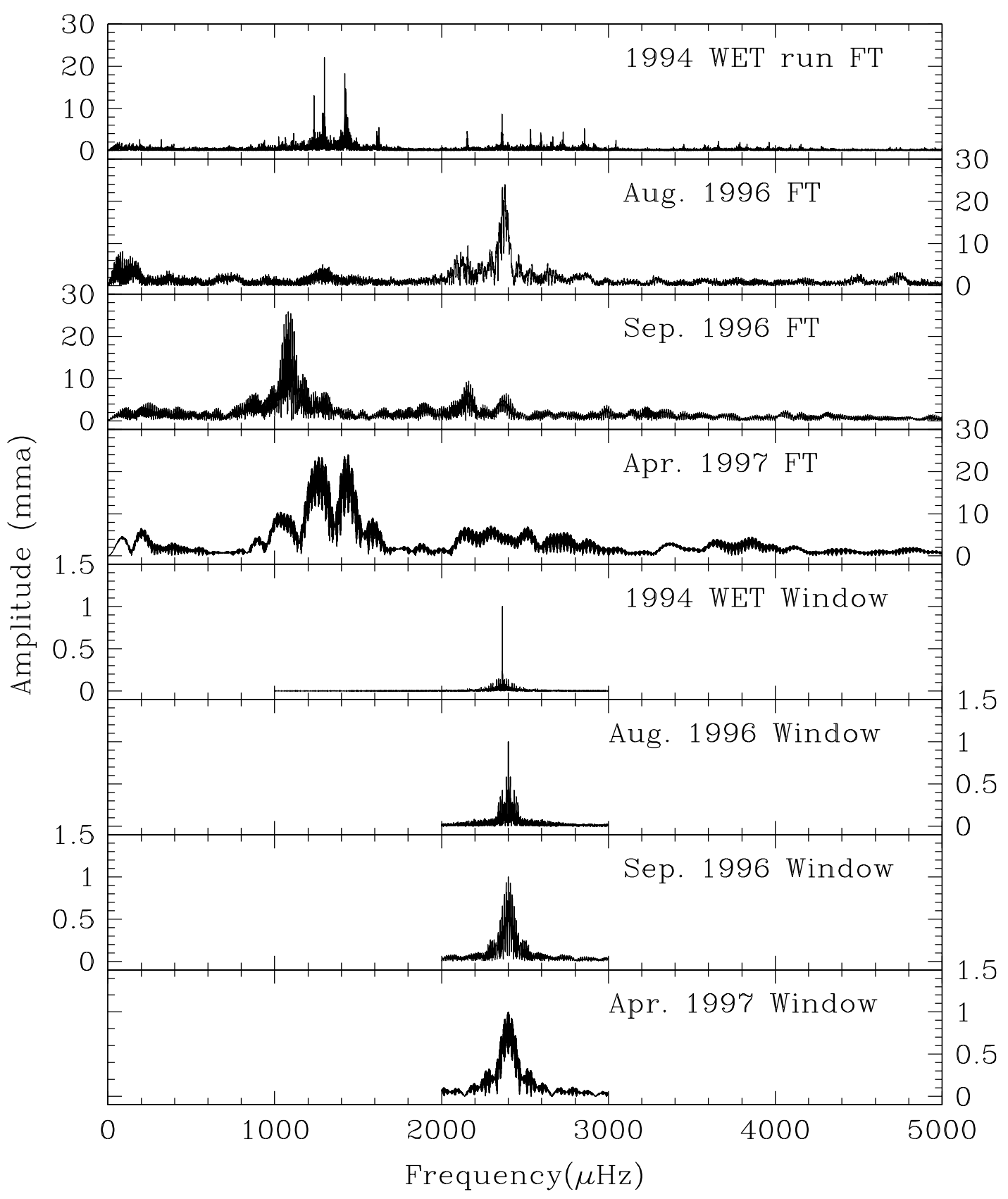

Fig. 7. GD 358 Fourier transform at four different times along with their spectral windows. The 1994 and 1997 Fourier transforms look similar (within the observed frequency resolution, that is). The September 1996 data look similar as well to these two data sets, but the highest amplitude modes have shorter frequencies (longer period). Obviously, the August 1996 Fourier transform looks very different from the other Fourier transforms.

correlated. The probability that a peak in the Fourier transform has a $1 / 1000$ chance of being due to noise, not a real signal, for our large frequency range of interest, ${ }^{1}$ requires at least peaks above $4\langle A m p\rangle$, where the average amplitude $\langle A m p\rangle$ is the square root of the power average (see also Breger et al. 1993 and Kuschnig et al. 1997 for a similar estimative).

Table 5 shows that the noise, represented by $\langle A m p\rangle$, for the 2000 data set is the smallest to date, allowing us to detect smaller amplitude peaks. Several peaks in the multi-frequency

\footnotetext{
${ }^{1}$ Corresponding to a number of multiple trials larger than the number of data points.
}

Table 5. Average amplitude of datasets, from 1000 to $3000 \mu \mathrm{Hz}$.

\begin{tabular}{ccc}
\hline \hline Year & BCT $_{\text {start }}$ & $\langle$ Amp $\rangle$ \\
\hline & (days) & $(\mathrm{mma})$ \\
1990 & 2448031.771867 & 0.62 \\
1994 & 2449475.001705 & 0.58 \\
1996 & 2450307.617884 & 1.44 \\
2000 & 2451702.402508 & 0.29 \\
\hline
\end{tabular}

fits are below the $4\langle\mathrm{Amp}\rangle$ limit and therefore should be considered only as upper limits to the components. 


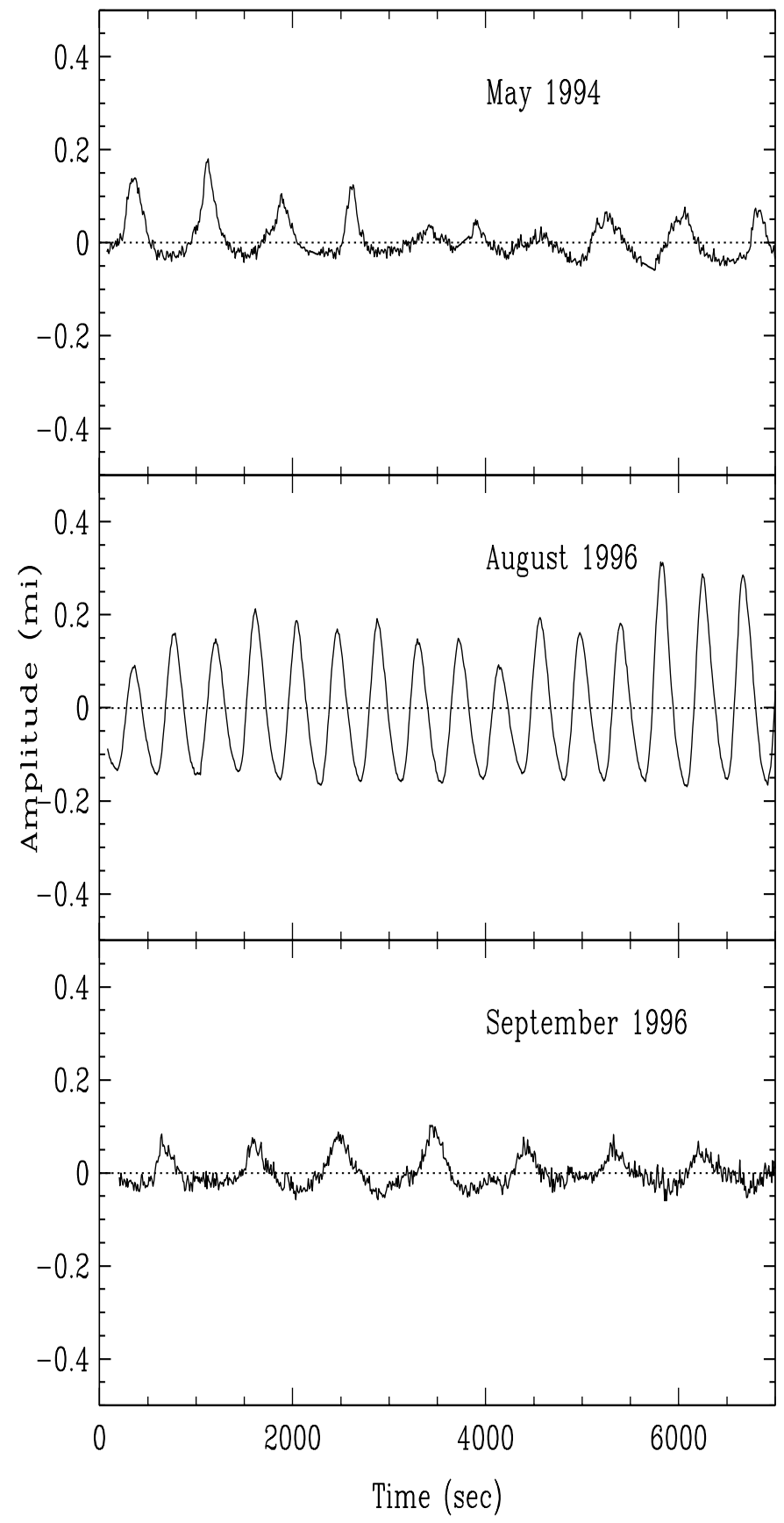

Fig. 8. GD 358 lightcurves over time. The shape of the lightcurve was sinusoidal when the amplitude was highest. The 1994 and September 1996 data exhibit similar pulse shapes and their corresponding power spectra also look similar (Fig. 7).

The present mode identification follows that of the 1990 data set, published by Winget et al. (1994). They represent the pulsations in terms of spherical harmonics $Y_{\ell, m}$, with each eigenmode described by three quantum numbers: the radial overtone number $k$, the degree $\ell$, also called the angular momentum quantum number, and the azimuthal number $m$, with $2 \ell+1$ possible values, from $-\ell$ to $+\ell$. For a perfectly spherical star, all $(2 \ell+1)$ eigenmodes with the same values of $k$ and $\ell$ should have the same frequency, but rotation causes each eigenmode to have a frequency also dependent on $m$. Magnetic fields
Table 6. Our multisinusoidal fit to the main periodicities in 1990.

\begin{tabular}{lcrr}
\hline \hline$k$ & $\begin{array}{c}\text { Frequency } \\
(\mu \mathrm{Hz})\end{array}$ & $\begin{array}{r}\text { Amplitude } \\
(\mathrm{mma})\end{array}$ & $\begin{array}{r}T_{\max } \\
(\mathrm{s})\end{array}$ \\
\hline 18 & $1233.408 \pm 0.017$ & $5.05 \pm 0.13$ & $731 \pm 7$ \\
$17^{+}$ & $1291.282 \pm 0.018$ & $5.04 \pm 0.14$ & $395 \pm 6$ \\
17 & $1297.590 \pm 0.006$ & $14.60 \pm 0.14$ & $24 \pm 2$ \\
$17^{-}$ & $1303.994 \pm 0.019$ & $4.71 \pm 0.14$ & $411 \pm 7$ \\
$16^{+}$ & $1355.664 \pm 0.106$ & $0.87 \pm 0.14$ & $471 \pm 37$ \\
16 & $1361.709 \pm 0.040$ & $2.21 \pm 0.14$ & $18 \pm 14$ \\
$16^{-}$ & $1368.568 \pm 0.031$ & $2.96 \pm 0.14$ & $627 \pm 11$ \\
$15^{+}$ & $1420.932 \pm 0.010$ & $9.32 \pm 0.14$ & $416 \pm 3$ \\
15 & $1427.402 \pm 0.005$ & $19.24 \pm 0.14$ & $425 \pm 2$ \\
$15^{-}$ & $1433.853 \pm 0.011$ & $7.90 \pm 0.14$ & $88 \pm 4$ \\
$14^{+}$ & $1513.023 \pm 0.017$ & $5.23 \pm 0.14$ & $123 \pm 5$ \\
14 & $1518.991 \pm 0.009$ & $9.71 \pm 0.14$ & $270 \pm 3$ \\
$14^{-}$ & $1525.873 \pm 0.016$ & $5.35 \pm 0.13$ & $459 \pm 5$ \\
$13^{+}$ & $1611.671 \pm 0.016$ & $5.70 \pm 0.14$ & $140 \pm 5$ \\
13 & $1617.297 \pm 0.017$ & $5.28 \pm 0.14$ & $508 \pm 5$ \\
$13^{-}$ & $1623.644 \pm 0.019$ & $4.70 \pm 0.14$ & $407 \pm 5$ \\
12 & $1733.850 \pm 0.163$ & $0.53 \pm 0.13$ & $474 \pm 44$ \\
$11^{+}$ & $1840.022 \pm 0.136$ & $0.65 \pm 0.14$ & $41 \pm 35$ \\
11 & $1846.247 \pm 0.135$ & $0.66 \pm 0.14$ & $504 \pm 34$ \\
$11^{-}$ & $1852.099 \pm 0.093$ & $0.94 \pm 0.14$ & $132 \pm 24$ \\
$10^{+}$ & $1994.240 \pm 1.071$ & $\leq 0.14$ & \\
10 & $1998.919 \pm 0.060$ & $1.50 \pm 0.14$ & $25 \pm 14$ \\
$10^{-}$ & $2007.992 \pm 0.117$ & $0.84 \pm 0.14$ & $7 \pm 26$ \\
$9^{+}$ & $2150.430 \pm 0.048$ & $1.932 \pm 0.13$ & $174 \pm 10$ \\
9 & $2154.052 \pm 0.020$ & $4.59 \pm 0.13$ & $336 \pm 4$ \\
$9^{-}$ & $2157.834 \pm 0.032$ & $2.81 \pm 0.13$ & $400 \pm 7$ \\
$8^{+}$ & $2358.975 \pm 0.016$ & $5.68 \pm 0.13$ & $120 \pm 3$ \\
8 & $2362.588 \pm 0.016$ & $5.77 \pm 0.13$ & $422 \pm 3$ \\
$8^{-}$ & $2366.418 \pm 0.017$ & $5.34 \pm 0.13$ & $268 \pm 3$ \\
\hline & & & \\
\hline
\end{tabular}

also lift the $m$ degeneracy. The assigned radial order $k$ value are the outcome of a comparison with model calculations presented in Bradley \& Winget (1994), and are consistent with the observed mass and parallax, as discussed in their paper. Vuille et al. (2000) determinations followed the above ones. In the upper part of Fig. 1, we placed a mark for equally spaced periods (correct in the asymptotic limit), using the $38.9 \mathrm{~s}$ spacing derived by Vuille et al., starting with the $k=17$ mode. The observed period spacings in the FT are very close to equal, consistent with previous observations.

\subsection{Nonlinear least squares results from 1990, 1994, and 1996}

For a more self-consistent comparison, we took the data from the 1990, 1994 WET runs and the August 1996 run and derived the periods of the dominant modes via a nonlinear least squares fit. In Tables 6, 7 and 8 we present the results of a non-linear simultaneous least squares fit of 23 to 29 sinusoids, representing the main periodicities, to the 1990, 1994 and 1996 data sets. We use the nomenclature $k^{a}$, for example $15^{-}$, to represent a subcomponent with $m=-1$ of the $k=15$ mode in these tables. 
Table 7. Main periodicities in 1994.

\begin{tabular}{lcrr}
\hline \hline$k$ & $\begin{array}{c}\text { Frequency } \\
(\mu \mathrm{Hz})\end{array}$ & $\begin{array}{r}\text { Amplitude } \\
(\mathrm{mma})\end{array}$ & $\begin{array}{r}T_{\max } \\
(\mathrm{s})\end{array}$ \\
\hline $18^{+}$ & $1228.712 \pm 0.022$ & $2.77 \pm 0.13$ & $252.6 \pm 12.0$ \\
18 & $1235.493 \pm 0.005$ & $12.94 \pm 0.13$ & $170.9 \pm 2.6$ \\
$18^{-}$ & $1242.364 \pm 0.016$ & $3.66 \pm 0.13$ & $62.4 \pm 9.0$ \\
$17^{+}$ & $1291.093 \pm 0.010$ & $6.17 \pm 0.13$ & $250.3 \pm 5.1$ \\
17 & $1297.741 \pm 0.003$ & $22.11 \pm 0.13$ & $37.7 \pm 1.4$ \\
$17^{-}$ & $1304.459 \pm 0.010$ & $6.25 \pm 0.13$ & $615.0 \pm 5.0$ \\
$16^{+}$ & $1355.388 \pm 0.035$ & $1.70 \pm 0.13$ & $167.0 \pm 17.6$ \\
16 & $1362.298 \pm 0.060$ & $<0.89$ & \\
$16^{-}$ & $1368.541 \pm 0.031$ & $1.92 \pm 0.13$ & $322.3 \pm 15.5$ \\
$15^{+}$ & $1419.650 \pm 0.003$ & $18.37 \pm 0.13$ & $46.4 \pm 1.6$ \\
15 & $1426.408 \pm 0.004$ & $15.55 \pm 0.13$ & $239.7 \pm 1.8$ \\
$15^{a}$ & $1430.879 \pm 0.006$ & $10.61 \pm 0.13$ & $187.9 \pm 2.7$ \\
$15^{-}$ & $1433.169 \pm 0.014$ & $4.46 \pm 0.13$ & $104.1 \pm 6.5$ \\
14 & $1519.903 \pm 0.028$ & $1.09 \pm 0.13$ & $485.2 \pm 20.0$ \\
$13^{+}$ & $1611.357 \pm 0.012$ & $5.02 \pm 0.13$ & $466.0 \pm 5.0$ \\
13 & $1617.474 \pm 0.009$ & $3.46 \pm 0.13$ & $183.3 \pm 1.1$ \\
$13^{-}$ & $1624.568 \pm 0.010$ & $6.07 \pm 0.13$ & $101.0 \pm 4.1$ \\
12 & $1746.766 \pm 0.064$ & $0.93 \pm 0.13$ & $414.2 \pm 25.0$ \\
11 & $1863.004 \pm 0.184$ & $<0.71$ & \\
10 & $2027.325 \pm 0.457$ & $<0.46$ & \\
$9^{+}$ & $2150.504 \pm 0.019$ & $3.15 \pm 0.13$ & $346.7 \pm 6.1$ \\
9 & $2154.124 \pm 0.013$ & $4.76 \pm 0.13$ & $12.3 \pm 4.0$ \\
$9^{-}$ & $2157.841 \pm 0.022$ & $2.69 \pm 0.13$ & $144.2 \pm 7.1$ \\
$8^{+}$ & $2358.883 \pm 0.013$ & $4.50 \pm 0.13$ & $398.2 \pm 3.8$ \\
8 & $2362.636 \pm 0.006$ & $9.25 \pm 0.13$ & $274.6 \pm 1.8$ \\
$8^{-}$ & $2366.508 \pm 0.007$ & $4.22 \pm 0.13$ & $169.7 \pm 3.8$ \\
\hline & & &
\end{tabular}

The difference in the frequencies reported in this paper compared to the previous ones is due to our use of the simultaneous non-linear least-squares frequency fitting rather than using the Fourier Transform frequencies.

We note that both the Fourier analysis and multi-sinusoidal fit assume the signal is composed of sinusoids with constant amplitudes, which is clearly violated in the 1996 data set. The changing amplitudes introduce spurious peaks in the Fourier transform. This will not affect the frequency of the modes, but the inferred amplitude will be a poor match to the (nonsinusoidal) light curve amplitude.

In Table 5 we present the average amplitude of the data sets, from 1000 to $3000 \mu \mathrm{Hz}$, after the main periodicities, all above $4\langle$ Amp $\rangle$, have been subtracted. For the 2000 data set, the initial $\langle\mathrm{Amp}\rangle$ for the frequency range from 0 to $10000 \mu \mathrm{Hz}$, is $0.69 \mathrm{mma}$. For the high frequency range above $3000 \mu \mathrm{Hz}$, $\langle\mathrm{Amp}\rangle \simeq 0.2 \mathrm{mma}$.

\subsection{Mode analysis of the 2000 WET data}

To provide the most accurate frequencies possible, we rely on a non-linear least squares fit of sinusoidal modes with guesses to the observed periods, since these better take into account contamination or slight frequency shifts due to aliasing. In Table 9 we present the results of a simultaneous non-linear least squares fit of 29 sinusoids, representing the main
Table 8. Main modes in 1996.

\begin{tabular}{lcrr}
\hline \hline$k$ & $\begin{array}{c}\text { Frequency } \\
(\mu \mathrm{Hz})\end{array}$ & $\begin{array}{r}\text { Amplitude } \\
(\mathrm{mma})\end{array}$ & $\begin{array}{r}T_{\max } \\
(\mathrm{s})\end{array}$ \\
\hline 19 & $1172.66 \pm 0.15$ & $2.5 \pm 0.6$ & $17.4 \pm 47.5$ \\
18 & $1253.65 \pm 1.01$ & $<2.1$ & \\
$17^{+}$ & $1291.13 \pm 0.11$ & $4.3 \pm 0.6$ & $653.6 \pm 28.1$ \\
$17^{0}$ & $1295.38 \pm 0.17$ & $2.6 \pm 0.6$ & $139.7 \pm 43.6$ \\
$17^{-}$ & $1304.68 \pm 0.11$ & $4.8 \pm 0.6$ & $346.3 \pm 25.7$ \\
$16^{+}$ & $1355.21 \pm 2.02$ & $<1.9$ & \\
$16^{0}$ & $1362.55 \pm 0.15$ & $2.7 \pm 0.6$ & $172.2 \pm 41.2$ \\
$16^{-}$ & $1379.64 \pm 0.16$ & $2.7 \pm 0.6$ & $378.6 \pm 41.0$ \\
$15^{0}$ & $1427.47 \pm 0.92$ & $<2.2$ & \\
$15^{-}$ & $1434.36 \pm 0.18$ & $2.2 \pm 0.6$ & $154.5 \pm 47.1$ \\
14 & $1520.58 \pm 0.18$ & $2.0 \pm 0.6$ & $398.3 \pm 46.6$ \\
$13^{+}$ & $1611.60 \pm 0.18$ & $2.1 \pm 0.6$ & $461.8 \pm 42.7$ \\
$13^{0}$ & $1617.51 \pm 0.35$ & $1.1 \pm 0.6$ & $183.5 \pm 79.9$ \\
$13^{-}$ & $1619.63 \pm 0.84$ & $<2.2$ & \\
12 & $1736.10 \pm 0.34$ & $1.1 \pm 0.6$ & $323.6 \pm 75.2$ \\
11 & $1862.93 \pm 0.39$ & $0.9 \pm 0.6$ & $58.8 \pm 78.7$ \\
10 & $2027.41 \pm 0.26$ & $1.4 \pm 0.6$ & $471.9 \pm 47.9$ \\
$9^{+}$ & $2149.97 \pm 0.07$ & $5.6 \pm 0.6$ & $69.8 \pm 11.8$ \\
$9^{0}$ & $2153.84 \pm 0.05$ & $7.6 \pm 0.6$ & $155.2 \pm 8.5$ \\
$9^{-}$ & $2157.89 \pm 0.04$ & $9.1 \pm 0.6$ & $426.7 \pm 7.2$ \\
$8^{+}$ & $2358.63 \pm 0.03$ & $12.6 \pm 0.6$ & $125.3 \pm 4.7$ \\
$8^{0}$ & $2362.50 \pm 0.02$ & $23.2 \pm 0.6$ & $104.8 \pm 2.5$ \\
$8^{-}$ & $2365.98 \pm 0.02$ & $22.2 \pm 0.6$ & $142.9 \pm 2.6$ \\
\hline & & &
\end{tabular}

periodicities of the 2000 data set, simultaneously. All the phases have been measured with respect to the barycentric Julian coordinated date BCT 2451702.402508.

Armed with the new frequencies in Table 9, we comment on regions of particular interest in the FT. First, we identify several newly detected modes at $P=373.76 \mathrm{~s}, f=2675.49 \mu \mathrm{Hz}$, amp $=8.43 \mathrm{mma} ; P=852.52 \mathrm{~s}, f=1172.99 \mu \mathrm{Hz}, \mathrm{amp}=$ $2.74 \mathrm{mma}$; and $P=900.13 \mathrm{~s}, f=1110.95 \mu \mathrm{Hz}$, amp $=$ $2.03 \mathrm{mma}$. Based on the mode assignments of Bradley \& Winget (1994) we identify these modes as $k=7,19$, and 20. The mode identification is based on the proximity of the detected modes with those predicted by the models, or even the asymptotical period spacings, but also because of resonant mode coupling, i.e., a stable mode will be driven to visibility if a coupled mode falls near its frequency, as it happens for $k=7$, which is very close to the combination of $k=17$ and $k=16$; and $k=20$, which falls near the resonance of the $8^{-}$and the $\ell=2$ mode at $1255 \mu \mathrm{Hz}$ (see next paragraph). It is important to note that these modes appear in combination peaks with other modes, as shown in Table 11. This reinforces our belief that these modes are physical modes, and not just erroneously identified combination peaks. We note that Bradley (2002) analyzed single site data taken over several years, and found peaks at 1172 or $1183 \mu \mathrm{Hz}$ in April 1985, May 1986, and June 1992 data, lending additional credence to the detection of the $k=19$ mode or its alias.

The first previously known region of interest surrounds the $k=18$ mode, which lies near $1233 \mu \mathrm{Hz}$, according to previous observations. In the 2000 data, the largest amplitude peak 
Table 9. Main modes in 2000.

\begin{tabular}{|c|c|c|c|c|}
\hline$k$ & $\begin{array}{c}\text { Frequency } \\
\quad(\mu \mathrm{Hz})\end{array}$ & $\begin{array}{r}\text { Period } \\
(\mathrm{s})\end{array}$ & $\begin{array}{r}\text { Amplitude } \\
\text { (mma) }\end{array}$ & $\begin{array}{r}T_{\max } \\
(\mathrm{s})\end{array}$ \\
\hline 20 & $1110.960 \pm 0.017$ & $900.122 \pm 0.014$ & $2.04 \pm 0.08$ & $870.19 \pm 8.61$ \\
\hline 19 & $1172.982 \pm 0.013$ & $852.528 \pm 0.009$ & $2.74 \pm 0.08$ & $164.86 \pm 6.07$ \\
\hline$\ell=2$ & $1255.400 \pm 0.002$ & $796.556 \pm 0.002$ & $14.86 \pm 0.08$ & $747.02 \pm 1.05$ \\
\hline 18 & $1233.595 \pm 0.018$ & $810.639 \pm 0.012$ & $1.96 \pm 0.08$ & $354.91 \pm 8.11$ \\
\hline $17^{+}$ & $1294.284 \pm 0.094$ & $772.628 \pm 0.056$ & $0.38 \pm 0.082$ & $579.63 \pm 39.54$ \\
\hline 17 & $1296.599 \pm 0.001$ & $771.248 \pm 0.001$ & $29.16 \pm 0.08$ & $247.81 \pm 0.52$ \\
\hline $17^{-}$ & $1301.653 \pm 0.053$ & $768.254 \pm 0.031$ & $0.68 \pm 0.08$ & $314.52 \pm 22.52$ \\
\hline 16 & $1362.238 \pm 0.159$ & $734.086 \pm 0.086$ & $0.42 \pm 0.12$ & $263.36 \pm 63.93$ \\
\hline $16^{-}$ & $1378.806 \pm 0.007$ & $725.265 \pm 0.004$ & $5.35 \pm 0.08$ & $514.70 \pm 2.66$ \\
\hline $15^{+}$ & $1420.095 \pm 0.001$ & $704.178 \pm 0.001$ & $29.69 \pm 0.08$ & $418.14 \pm 0.49$ \\
\hline 15 & $1428.090 \pm 0.052$ & $700.236 \pm 0.025$ & $0.70 \pm 0.08$ & $217.29 \pm 19.78$ \\
\hline $15^{-}$ & $1432.211 \pm 0.036$ & $698.221 \pm 0.018$ & $1.08 \pm 0.089$ & $355.58 \pm 13.30$ \\
\hline 14 & $1519.811 \pm 0.134$ & $657.977 \pm 0.058$ & $0.266 \pm 0.08$ & $301.55 \pm 48.28$ \\
\hline $13^{+}$ & $1611.084 \pm 0.116$ & $620.700 \pm 0.045$ & $0.31 \pm 0.08$ & $448.66 \pm 39.81$ \\
\hline 13 & $1617.633 \pm 0.174$ & $618.187 \pm 0.066$ & $0.21 \pm 0.08$ & $448.19 \pm 58.97$ \\
\hline $13^{-}$ & $1625.170 \pm 0.235$ & $615.320 \pm 0.089$ & $0.15 \pm 0.08$ & $299.24 \pm 79.32$ \\
\hline 12 & $1736.277 \pm 0.034$ & $575.945 \pm 0.011$ & $1.04 \pm 0.08$ & $230.37 \pm 10.79$ \\
\hline 11 & $1862.871 \pm 0.042$ & $536.806 \pm 0.012$ & $0.84 \pm 0.08$ & $503.66 \pm 12.43$ \\
\hline 10 & $2027.008 \pm 0.028$ & $493.338 \pm 0.007$ & $1.29 \pm 0.08$ & $350.14 \pm 7.49$ \\
\hline $9^{+}$ & $2150.462 \pm 0.012$ & $465.016 \pm 0.003$ & $2.96 \pm 0.08$ & $35.49 \pm 3.09$ \\
\hline 9 & $2154.021 \pm 0.007$ & $464.248 \pm 0.001$ & $5.34 \pm 0.08$ & $252.38 \pm 1.71$ \\
\hline $9^{-}$ & $2157.731 \pm 0.014$ & $463.450 \pm 0.003$ & $2.57 \pm 0.08$ & $174.68 \pm 3.53$ \\
\hline $8^{+}$ & $2359.119 \pm 0.006$ & $423.887 \pm 0.001$ & $5.57 \pm 0.08$ & $166.60 \pm 1.49$ \\
\hline 8 & $2362.948 \pm 0.094$ & $423.200 \pm 0.017$ & $0.38 \pm 0.08$ & $81.50 \pm 21.93$ \\
\hline $8^{-}$ & $2366.266 \pm 0.006$ & $422.607 \pm 0.001$ & $5.79 \pm 0.08$ & $418.36 \pm 1.44$ \\
\hline $2 \times 18$ & $2510.761 \pm 0.021$ & $398.286 \pm 0.003$ & $1.70 \pm 0.08$ & $334.47 \pm 4.58$ \\
\hline $2 \times 17$ & $2593.208 \pm 0.004$ & $385.623 \pm 0.001$ & $7.82 \pm 0.08$ & $249.57 \pm 0.96$ \\
\hline 7 & $2675.487 \pm 0.004$ & $373.764 \pm 0.001$ & $8.49 \pm 0.08$ & $193.30 \pm 0.86$ \\
\hline $2 \times 15$ & $2840.195 \pm 0.008$ & $352.089 \pm 0.001$ & $4.29 \pm 0.08$ & $47.50 \pm 1.60$ \\
\hline
\end{tabular}

in this region lies at $1255 \mu \mathrm{Hz}$, which is over $20 \mu \mathrm{Hz}$ from the previous location. Given that other modes (especially the one at $k=17$ ) has shifted by less than $4 \mu \mathrm{Hz}$, we are inclined to rule out the possibility that the $k=18$ mode shifted by $20 \mu \mathrm{Hz}$. One possible solution is offered by seismological models of GD 358, which predict an $\ell=2$ mode near $1255 \mu \mathrm{Hz}$. For example, the best ML2 fit to the 1990 data (from Metcalfe et al. 2002, Table 3), has an $\ell=2$ mode at $1252.6 \mu \mathrm{Hz}(P=$ $798.3 \mathrm{~s}$ ). This would also be consistent with the larger number of subcomponents detected, although they may be caused only by amplitude changes during the observations. Figure 9 shows the region of the $k=18$ mode in the FT for the 1990 data set (solid) and the 2000 data set (dashed); it is consistent with the $k=18$ mode being the $1233 \mu \mathrm{Hz}$ for both data sets, and they even have similar amplitudes. While we avoided having to provide an explanation for why only the $k=18$ mode would shift by $20 \mu \mathrm{Hz}$, we have introduced another problem, as geometrical cancellation for an $\ell=2$ mode introduces a factor of 0.26 in relation to unity for an $\ell=1$ mode. Thus, the identification of the $1255 \mu \mathrm{Hz}$ as an $\ell=2$ mode, which has a measured amplitude of $14.86 \mathrm{mma}$, implies a physical amplitude higher than that of the highest amplitude $\ell=1$ mode, around $30 \mathrm{mma}$.
Kotak et al. (2003), analyzing time-resolved spectra obtained at the Keck in 1998, show the velocity variations of the $k=18$ mode at $1233 \mu \mathrm{Hz}$ is similar to those for the $k=15$ and $k=17$ modes, concluding all modes are $\ell=1$. They did not detect a mode at $1255 \mu \mathrm{Hz}$.

In Fig. 10 we show the pre-whitened results; pre-whitening was done by subtracting from the observed light curve a synthetic light curve constructed with a single sinusoid with frequency, amplitude and phase that minimizes the Fourier spectrum at the frequency of the highest peak. A new Fourier spectrum is calculated and the next dominant frequency is subtracted, repeating the procedure until no significant power is left. It is important to notice that with pre-whitening, the order of subtraction matters. As an example, in the 2000 data set, if we subtract the largest peak in the region of the $k=18$ mode, at $1255.41 \mu \mathrm{Hz}$, followed by the next highest peak at $1256.26 \mu \mathrm{Hz}$ and the next at $1254.44 \mu \mathrm{Hz}$, we are left with a peak at only $1.3 \mathrm{mma}$ at $1232.76 \mu \mathrm{Hz}$. But if instead we subtract only the $1255.41 \mu \mathrm{Hz}$ followed by the peak left at $1233.24 \mu \mathrm{Hz}$, its amplitude is around $3.1 \mathrm{mma}$, i.e., larger. Prewhitening assumes the frequencies are independent in the observed, finite, data set. If they were, the order of subtraction 


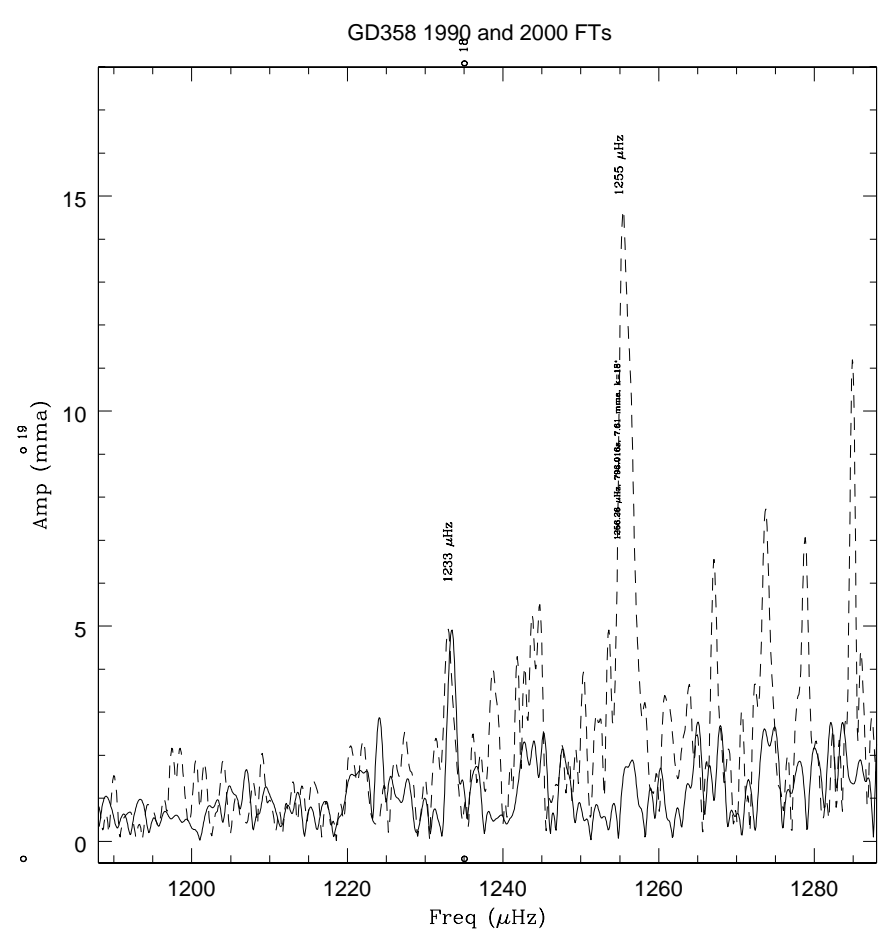

Fig. 9. Peaks around $k=18$ in the 1990 (solid line) and 2000 (dashed line) transforms.

would not affect the result. Because the order of subtraction matters, the basic assumption of pre-whitening does not apply. We attempt to minimize this effect by noting that the frequencies change less than the amplitudes, and use the FT frequencies in a simultaneous non-linear least squares fit of all the eigenmode frequencies. But even the simultaneous non-linear least squares fit uses the values of the Fourier transform as starting points, and could converge to a local minimum of the variance instead of the global minimum.

The modes with periods between 770 and $518 \mathrm{~s}(k=17$ through 13) are present in the 2000 data, though with different amplitudes than in previous years. Another striking feature of the peaks in 2000 is that one multiplet member of each mode has by far the largest amplitude, so that without data from previous WET runs, we would not know that the modes are rotationally split. The frequencies of these modes are stable to about $1 \mu \mathrm{Hz}$ or less with the exception of the $16^{-}$mode, where the frequency jumped from about $1368.5 \mu \mathrm{Hz}$ in 1990 and 1994 to about $1379 \mu \mathrm{Hz}$ in 1996 and 2000 (see Fig. 11). Most of these frequency changes are larger than the formal frequency uncertainty from a given run (typically less than $0.05 \mu \mathrm{Hz}$ ), so there is some process in GD 358 that causes the mode frequencies to "wobble" from one run to the next. We speculate that this may be related to non-linear mode coupling effects. Whatever the origin of the frequency shifts, it renders these modes useless for studying evolutionary timescales through rates of period change.

The $k=12$ through 10 modes deserve separate mention because their amplitudes are always small; between 1990 and 2000, the largest amplitude peak was only $1.6 \mathrm{mma}$. The small amplitudes can make accurate frequency determinations difficult, and all three modes have frequency shifts of 13 to $33 \mu \mathrm{Hz}$ between the largest amplitude peaks in a given mode. The $k=10$ mode shows the largest change with the 1990 data showing the largest peaks at $1998.7 \mu \mathrm{Hz}$ and $2008.2 \mu \mathrm{Hz}$, while the 2000 data has one peak dominating the region at $2027.0 \mu \mathrm{Hz}$. An examination of the data in Bradley (2002) shows that the $k=12$ mode seems to consistently show a peak near 1733 to $1736 \mu \mathrm{Hz}$, and that only the 1994 data has the peak shifted to $1746.8 \mu \mathrm{Hz}$, suggesting that 1994 data may have found an alias peak or that the $1736 \mu \mathrm{Hz}$ mode could be the $m=+1$ member and the $1746.8 \mu \mathrm{Hz}$ mode is the $m=-1$ member. The data in Bradley (2002) do not show convincing evidence for the $k=11$ or 10 modes, so we cannot say anything else about them.

It is interesting to note that the $k=8$ and $k=9$ modes are always seen as a triplet, with $3.58 \mu \mathrm{Hz}$ separation for $k=$ 9 , even in the 1996 data set. Our measured spacings are 3.54 and $3.69 \mu \mathrm{Hz}$, from $m=-1$ to $m=0$ and $m=0$ to $m=1$. The $k=8$ mode in 2000 shows an $\mathrm{m}=0$ component below our statistical detection limit $(A=0.41 \mathrm{mma}$, when the local $\langle$ Amp $\rangle=0.29 \mathrm{mma}$ ), but the $m=1$ and $m=-1$ modes remain separated by $2 \times 3.58 \mu \mathrm{Hz}$. All the higher $k$ modes are seen as singlets in the 2000 data set. We also note that the $k=8$ and 9 modes have by far the most stable frequencies. The frequencies are always the same to within $0.3 \mu \mathrm{Hz}$, and in some cases better than $0.1 \mu \mathrm{Hz}$. However, the frequency shifts are large enough to mask any possible signs of evolutionary period change, as Fig. 12 shows. Thus, we are forced to conclude that GD 358 is not a stable enough "clock" to discern evolutionary rates of period change.

\section{Multiplet splittings}

As pointed out by Winget et al. (1994) and Vuille et al. (2000), the observed triplets in the 1900 and 1994 data sets had splittings ranging from $6.5 \mu \mathrm{Hz}$ from the "external" modes (such as $k=17$ ) to $3.6 \mu \mathrm{Hz}$ for the "internal" modes $k=8$ and 9 . Winget et al. interpreted these splittings to be the result of radial differential rotation, and Kawaler et al. (1999) examined this interpretation in more detail. An examination of the frequencies found in the 2000 data set, shown in Table 9, shows that the multiplet structure is much harder to discern, since the $k=10$ through 20 modes typically have only one multiplet member with a large amplitude. The obvious multiplet members have frequencies that agree with the 1990 data, except for the $16^{-}$mode, where there is a $+10.234 \mu \mathrm{Hz}$ shift in the 2000 data.

Note that the $k=10$ mode identified at $2027 \mu \mathrm{Hz}$ is different than the $1994 \mu \mathrm{Hz}$ identified by Winget et al. (1994) in the 1990 data. However, the peak they identified is not the highest peak in that region of the Fourier transform (see Fig. 13). Our analysis of the 1990 data has statistically significant $k=10$ peaks close to $1999 \mu \mathrm{Hz}$ and $2008 \mu \mathrm{Hz}$.

The only modes with obvious multiplet structure are the $k=9$ mode, which still shows an obvious $3.6 \mu \mathrm{Hz}$ split triplet, and the $k=8$ mode, which shows two peaks that are consistent with $2 \times 3.6 \mu \mathrm{Hz}$ separation.

In Table 11 we have a peak $3.3 \mu \mathrm{Hz}$ from the $k=15$, $m=1$ mode that we have not seen before; we call it the $15^{b}$ 


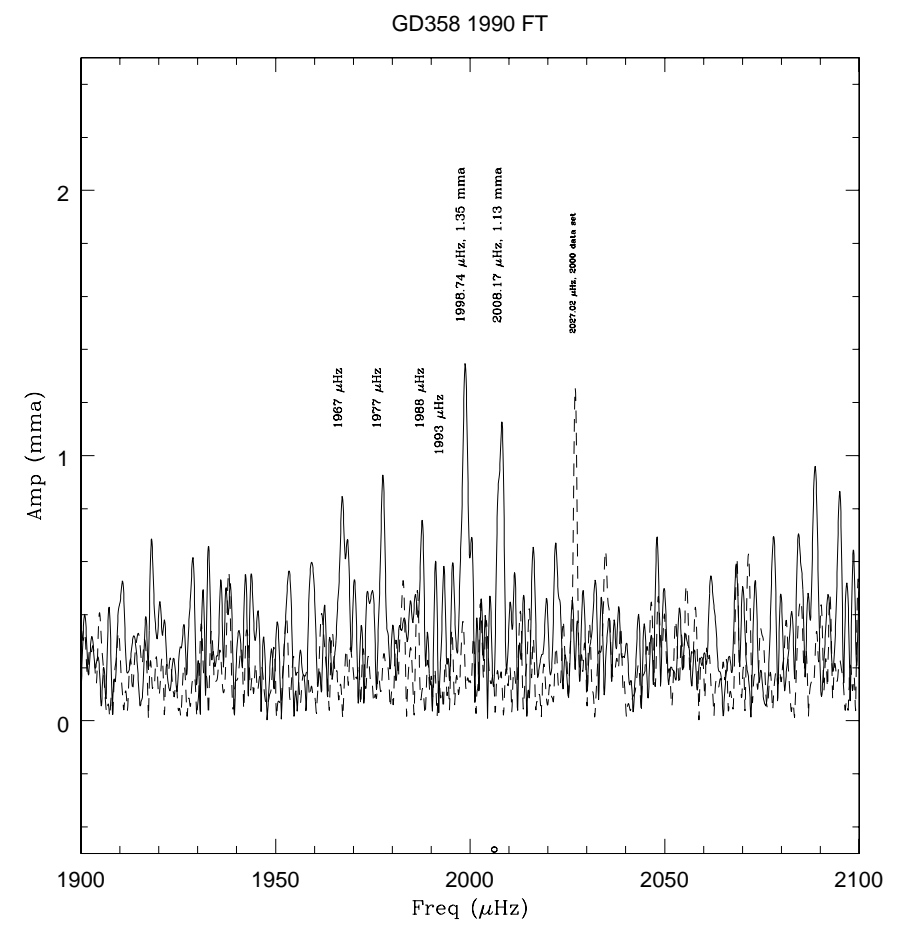

Fig. 13. Peaks around $k=10$ in the 1990 (solid line) and 2000 (dashed line) transforms.

mode. We are not certain whether this is another member of the $k=15$ multiplet (analogous to the $15^{a}$ mode in the 1994 WET data) or if it is something else. The 1994 data set also presented a large peak $4.4 \mu \mathrm{Hz}$ from $k=15, m=0$, which we call the $15^{a}$ mode, in addition to the $m= \pm 1$ components. We have not seen this $15^{a}$ mode in any other data set other than the 1994 WET run. The identity of the "extra subcomponents" remains an unsolved mystery.

\section{Linear combinations}

Winget et al. (1994) and Vuille et al. (2000) show that most of the periodicities are in fact linear combination peaks of the main peaks (eigenmodes). Combination peaks are what we call peaks in the FT whose frequencies are equal to the sum or difference of two (or more) the the $\ell=1$ or 2 mode frequencies. The criteria for selection of the combination peaks was that the frequency difference between the combination peak and the sum of the "parent mode" frequencies must be smaller than our resolution, which is typically around $1 \mu \mathrm{Hz}$. The last column of Table 11 shows the frequency difference.

For example, only 28 of the more than 180 peaks in Winget et al. (1994) are $\ell=1$ modes; the rest are combination peaks up to third order (i.e., three modes are involved). The $\ell=1$ modes lie in the region 1000 to $2500 \mu \mathrm{Hz}$, and are identified as modes $k=18$ to 8 . In the 1994 data set analyzed by Vuille et al., combination peaks up to 4 th order were detected. In the 2000 data set we identify combination peaks up to 6th order, and most if not all remaining peaks are in fact linear combination peaks, as demonstrated in Table 11 and is shown in the pre-whitened FT of the 2000 data (see Fig. 14). Here too, we use the nomenclature $k^{a}$, for example $15^{-}$, to represent a subcomponent with $m=-1$ of the $k=15$ mode.
The so-called $\ell=2$ mode at $1255 \mu \mathrm{Hz}$, as well as $k=17$ and $k=15$ modes, have subcomponents, but probably they are not different $m$ value components, and are caused, most likely, by amplitude modulation. We say this because the frequency splittings are drastically different than in previous data, and for the $\ell=2$ mode, there are more than 5 possible subcomponent peaks present. We did not do an exhaustive search for all of the possible combination peaks up to 6th order in the Fourier transform, as we only took into account the peaks that had a probability smaller than $1 / 1000$ of being due to noise, and studied if they could be explained as combination peaks.

Brickhill's (1992) pulsation-convection interaction model predicts, and the observations reported by Winget et al. and Vuille et al. agree, that a combination peak involving two different modes always has a larger relative amplitude than a combination involving twice the frequency of a given mode (also called a harmonic peak). Wu's (2001) analytical expression leads to a factor of $1 / 2$ difference between a combination peak with two modes versus a harmonic peak, assuming that the amplitudes of both eigenmodes are the same. Vuille et al. claim that the relatively small amplitude of the $k=13$ mode in 1994 is affected by destructive beating of the nonlinear peak $(2 \times 15-18)$ and that the $k=16$ mode amplitude is affected by the $(15+18-17)$ combination peak. It is noteworthy that the peak at $1423.62 \mu \mathrm{Hz}$ is only $3.52 \mu \mathrm{Hz}$ from $k=15$, so it might be the $15^{-}$mode. However, the previously identified $15^{-}$was $6.7 \mu \mathrm{Hz}$ from it, and we consider the $1423.62 \mu \mathrm{Hz}$ peak to be either a result of amplitude modulation of the $k=15$ mode or yet another combination peak.

We note that the wealth of combination peaks and their relative amplitude offers insight into the amplitude limiting mechanism and would be worthy of the considerable theoretical and numerical effort required to understand it.

\section{Model-fitting with a genetic algorithm}

One of the major goals of our observations of GD 358 was to discover additional modes to help refine our seismological model fits. We were also interested in how much the globally optimal model parameters would change due to the slight shifts in the observed periods. With these goals in mind, we repeated the global model-fitting procedure of Metcalfe, Winget \& Charbonneau (2001) on several subsets of the new observations.

Our model-fitting method uses the parallel genetic algorithm described by Metcalfe \& Charbonneau (2003) to minimize the root-mean-square (rms) differences between the observed and calculated periods $\left(P_{k}\right)$ and period spacings $(\Delta P \equiv$ $\left.P_{k+1}-P_{k}\right)$ for models with effective temperatures $\left(T_{\text {eff }}\right)$ between 20000 and $30000 \mathrm{~K}$, total stellar masses $\left(M_{*}\right)$ between 0.45 and $0.95 M_{\odot}$, helium layer masses with $-\log \left(M_{\mathrm{He}} / M_{*}\right)$ between 2.0 and $\sim 7.0$, and an internal $\mathrm{C} / \mathrm{O}$ profile with a constant oxygen mass fraction $\left(X_{\mathrm{O}}\right)$ out to some fractional mass (q) where it then decreases linearly in mass to zero oxygen at $0.95 \mathrm{~m} / \mathrm{M}_{*}$. This technique has been shown to find the globally optimal set of parameters consistently among the many possible combinations in the search space, but it requires 
FT of GD358 data 2000
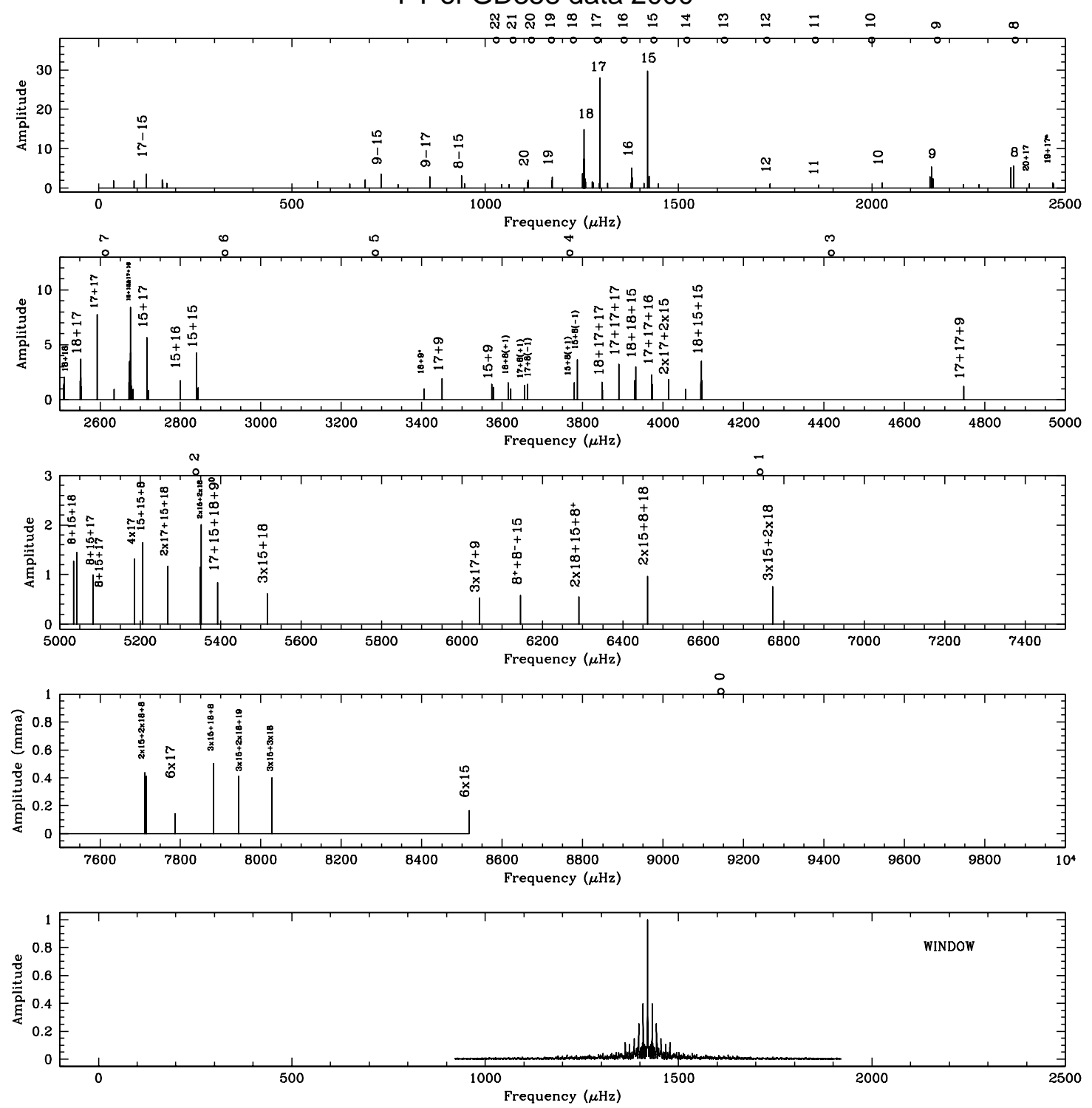

Fig. 14. Pre-whitened peaks in the 2000 transform.

between 200 and 4000 times fewer model evaluations than an exhaustive search of the parameter-space to accomplish this, and has a failure rate $<10^{-5}$.

We attempted to fit the 13 periods and period spacings defined by the $m=0$ components of the 14 modes identified as $k=7$ to $k=20$ in Table 9 . Because of our uncertainty about the proper identification of $k=18$ (see Sect. 4) we performed fits under two different assumptions: for Fit $a$ we assumed that the frequency near $1233 \mu \mathrm{Hz}$ was $k=18$ (similar to the frequency identified in 1990), and for Fit $b$ we assumed that the larger amplitude frequency near $1255 \mu \mathrm{Hz}$ was $k=18$. The results of these two fits led us to prefer the identification for $k=18$ in Fit $a$, and we included this in an additional fit using only the 11 modes from $k=8$ to $k=18$, which correspond to those identified in 1990 (Fit $c$ ). We performed an additional fit (Fit $d$ ) that included the same 13 periods used for Fit $a$, but ignored the period spacings. The optimal values for the five model parameters, and the root-mean-square residuals between the observed
Table 10. Optimal Fits to 2000 data.

\begin{tabular}{rrrrr}
\hline \hline Parameter & Fit $a$ & Fit $b$ & Fit $c$ & Fit $d$ \\
\hline \hline$T_{\text {eff }}(K)$ & 24300 & 23500 & 24500 & 22700 \\
$M_{*}\left(M_{\odot}\right)$ & 0.61 & 0.60 & 0.625 & 0.630 \\
$\log \left(M_{\mathrm{He}} / M_{*}\right)$ & -2.79 & -5.13 & -2.58 & -4.07 \\
$X_{\mathrm{O}}$ & 0.81 & 0.99 & 0.39 & 0.37 \\
$q\left(m / M_{*}\right)$ & 0.47 & 0.47 & 0.83 & 0.42 \\
$\sigma_{P}(s)$ & 2.60 & 3.65 & 2.12 & 1.72 \\
$\sigma_{\Delta P}(s)$ & 4.07 & 4.92 & 2.21 & $\ldots$ \\
\hline
\end{tabular}

and computed periods $\left(\sigma_{P}\right)$ and period spacings $\left(\sigma_{\Delta P}\right)$ for the four fits are shown in Table 10.

Our preferred solution from Table 10 is Fit $a$, because it includes our favored identification for the $k=18$ mode and the additional pulsation periods. The larger $\sigma_{\Delta P}$ in Fit $a$ compared to Fit $c$ is dominated by the large period spacings between the 
$k=19$ and 20 modes (47.6 s) and the $k=7$ and 8 modes $(49.4 \mathrm{~s})$. Fit $a$ has a mass and effective temperature that are essentially the same as the fit of Bradley \& Winget (1994), and are consistent with the spectroscopic values derived by Beauchamp et al. (1999). The other structural parameters are otherwise similar to those found by Metcalfe et al. (2001) $\left(T_{\text {eff }}=22600 \mathrm{~K}, M_{*}=0.650 M_{\odot}, \log \left(M_{\mathrm{He}} / M_{*}\right)=-2.74\right.$, $X_{\mathrm{O}}=0.84$, and $\left.q=0.49\right)$. We caution, however, that the large values of $\sigma_{P}$ and $\sigma_{\Delta P}$ for Fit $a$ imply that our model may not be an adequate representation of the real white dwarf star. New and unmodeled physical circumstances may have arisen between 1994 and 2000 (e.g. whatever caused the forte in 1996), which may account for the diminished capacity of our simple model to match the observed periods.

\section{Summary of observational clues}

Before embarking on our discussion, we recap the highlights of our observations. First, the 2000 WET data shows eigenmodes from at least $k=8$ through $k=19$. We may also have detected the $k=7$ and $k=20$ modes. However, their frequencies are similar to that of unrelated combination peaks, so their identification is less secure. For the first time, we have also found an $\ell=2$ mode in the GD 358 data; it is at $1255.4 \mu \mathrm{Hz}$. Second, we see relatively few multiplet modes for a given $k$, with the exception of the $k=8$ and 9 modes. While the multiplet structure of the $\ell=1$ modes is muted, the combination peaks are enhanced to the point that we see combination modes up to 6th order. Combined with the previous WET runs, we see evidence for anticorrelation between the presence of multiplet structure and combination peaks. The presence of amplitude variability of the $\ell=1$ mode continues. In the August 1996 data, we saw the most extreme example yet, where all of the observed light was in a single ( $k=8$ mode) for one night (which we call the "forte"). Data before and after the run show power in the nights before and after in other pulsation modes besides the $k=8$ and a much lower amplitude. The periods from the 1996 data are consistent with the 2000 data set, although there are differences in the details.

\section{Discussion and puzzles}

Using the $k=7,19$, and 20 modes in seismological fits produces a best-fitting model that is similar to that derived from only the $k=8$ through 18 modes, indicating that the new modes do not deviate drastically from the expected mode pattern.

The reappearance of modes with frequencies similar to those obtained before the mode disappeared (true of all modes from $k=8$ through 19), shows that the stellar structure sampled by these modes remained the same for almost 20 years. This is in spite of rapid amplitude change events like the "forte" one observed in August 1996. Our observations, coupled with guidance from the available theories of Brickhill (1992) and $\mathrm{Wu} \&$ Goldreich (2001) suggest that the "forte" event was probably an extreme manifestation of a nonlinear mode-coupling event that did not materially affect the structure of the star other than possibly the driving region. The appearance and disappearance of modes is similar to the behavior observed in the ZZ Ceti star
G 29-38 by Kleinman et al. (1998), and we note that "ensemble" seismology works for GD 358 as well as for the cool ZZ Ceti stars. The one caveat is that the $\sim 1 \mu \mathrm{Hz}$ frequency "wobbles" will place a limit on the accuracy of the seismology.

We also appear to have discovered an $\ell=2$ mode (at $1255.4 \mu \mathrm{Hz}$ ) in GD 358 for the first time, based on the match of the observed period to that of $\ell=2$ modes from our best fitting model. Our model indicates that this is the $k=34$ mode. This mode has a relatively large amplitude of $14.9 \mathrm{mma}$, which combined with the increased geometric cancellation (about $3.8 \times$ ) of an $\ell=2$ mode, implies that it has the largest amplitude of any mode observed in 2000 . We note the existence of several linear combination peaks involving the $1255 \mu \mathrm{Hz}$ mode, that also show complex structures. This lends credence to the $1255 \mu \mathrm{Hz}$ mode being a real mode, and that the complex structure is associated with the real mode (such as amplitude modulation), as opposed to being some sort of combination peak. The amplitude of the $1255 \mu \mathrm{Hz}$ mode changed during the WET run, so we suspect that the many subcomponents observed are most likely due to amplitude modulation.

The period structure of the 1990 and 1994 WET data sets are similar, but show that the amplitude of the modes, and even the fine structure, changes with time. In August 1996, the period structure changed rapidly and dramatically, with essentially all the observed pulsation power going to the $k=8$ mode. In spite of the large amplitude, the light curve was surprisingly sinusoidal, with a small contribution from the $k=9$ mode. Single site observations one month earlier (June 1996) and one month later (September 1996) show a period structure similar to those present in the 1990 and 1994 data sets. For the 2000 data set, the period structure shows close to equal frequency splittings, and the fine structure is different than observed before. Only the $k=9$ mode show the same clear triplet observed in 1990 and 1994, with the same frequency splitting. The $k=8$ mode shows the $m=-1$ and $m=1$ modes, while the central $m=0$ mode is below our $4\langle$ Amp $\rangle$ significance level. The other modes do not show clearly the triplet structure previously observed. The 1990 and 1994 data sets show the m-splitting expected by rotational splitting, but the change of the splitting frequency difference from $6 \mu \mathrm{Hz}$ to $3 \mu \mathrm{Hz}$ from $k=17$ to $k=8$ was interpreted as indicating differential rotation.

The apparent anticorrelation between the abundance of multiplet structure and the highest order of combination frequencies seen is a puzzle. As we do not expect the differential rotation profile of GD 358 changed in the last 10 years (and the splittings we do see in 2000 support this contention), the anticorrelation must be telling something about what is going on with rotation in the convection zone. We say this because the combination peaks are believed to be caused by the nonlinear response of the depth varying convection zone, and thus the increased order of combination peaks implies that the convection zone is more "efficient" at mixing eigenmodes to observable amplitudes. The $k=8$ and 9 modes continue to show obvious multiplet structure and little, if any, change in splitting. These modes are the most "internal" of the observed modes of GD 358, and we speculate that this must have some bearing on their multiplet structure's ability to persist. We do not see any obvious pattern in the dominant amplitude multiplet member 
with overtone number, so there is not an obvious pattern of rotational coupling to the convection zone for determining mode amplitude. We will need theoretical guidance to make sense of these observations.

A related puzzle is the presence of extra multiplet members and/or apparent large frequency shifts of modes in the $k=15$ and 16 multiplets. The $k=15$ mode shows an extra component at $1430.88 \mu \mathrm{Hz}$ in the 1994 data and a peak at $1423.62 \mu \mathrm{Hz}$ in the 2000 data that have not been seen before or since. Some possible explanations include: rapid amplitude modulation of a $k=15$ multiplet member that the FT interprets as an extra peak; the 2000 peak is about the right frequency to be another $\ell=2$ mode, if we use the $1255.4 \mu \mathrm{Hz}$ mode as a reference point; it could be an unattributed combination peak involving sums and differences of known modes; or it could be something else entirely. The large peak at about $1379 \mu \mathrm{Hz}$ in 1996 and 2000 is also something of a mystery. It is possible that the $k=16, m=-1$ component really changed by $10 \mu \mathrm{Hz}$ from the $1368 \mu \mathrm{Hz}$ observed in 1990, although we would have to explain why only this large amplitude multiplet member suffered this large a frequency change. Other possibilities include: the peak is a 1 cycle per day alias of another mode; the peak is a combination peak - the combination $15+(\ell=2)-17$ is a perfect frequency match; or possibly an $\ell=2$ mode, based on period spacing arguments. Further observations, data analysis with tools like wavelet analysis, and further model fitting may help determine which explanation fits the data best.

Brickhill (1992) proposed that the combination frequencies result from mixing of the eigenmode signals by a depthvarying surface convection zone when undergoing pulsation. He pointed out that the convective turnover time in DA and DB variable white dwarf stars occurs on a timescale much shorter than the pulsation period. As a consequence, the convective region adjusts almost instantaneously during a pulsation cycle. Brickhill demonstrated that the flux leaving the convective zone depends on the depth of the convective zone, which changes during the pulsation cycle, distorting the observed flux. This distortion introduces combination frequencies, even if the pulsation at the bottom of the convection zone is linear, i.e., a single sinusoidal frequency. Wu (2001) analytically calculated the amplitude and phases expected of such combination frequencies, and concluded that the convective induced distortion was roughly in agreement with GD358's 1994 observations, provided that the inclination of the pulsation axis to the line of sight is between $40^{\circ}$ and $50^{\circ}$. Wu also calculated that the harmonics for $\ell=2$ modes should be much higher than for $\ell=1$. However the theory overpredicts the amplitude of the $\ell=1$ harmonics. She also predicts that geometrical cancellation will, in principle, allow a determination of $\ell$ if both frequencies sums and differences are observed. These predictions still need testing.

While Wu \& Goldreich (2001) discuss parametric instability mechanisms for the amplitude of the pulsation modes, they only discuss the case where the parent mode is unstable and the daughter modes are stable. However, with GD 358, we have a different situation. The highest frequency $k=8$ and 9 modes can have as a daughter mode one of the lower frequency $(k=17,18$, or 19) $\ell=1$ modes and an $\ell=$ higher mode.
One or both or these daughter modes are actually pulsationally unstable as well, which we believe would require coupling to still lower frequency granddaughter modes that are predicted to be stable by our models and the calculations of Brickhill (1990, 1991) and Goldreich \& Wu (1999a,b). We suggest that occasionally the nonlinear coupling of the granddaughter and daughter modes with the $k=8$ and 9 modes can allow the $k=8$ and 9 modes to suffer abrupt amplitude changes when everything is "just right". In the meantime, the granddaughter modes will couple to the excited daughter modes $(k=13$ through 19 in general) to produce the observed amplitude instability of these modes. We need a quantitative theoretical treatment of this circumstance worked out to see if the predicted behavior matches what we observe in GD 358.

Observations of GD 358 have been both rewarding and vexing. We have been rewarded with enough $\ell=1$ modes being present to decipher the mode structure and perform increasingly refined asteroseismology of this star, starting with Bradley \& Winget (1994) up to the latest paper of Metcalfe et al. (2002). One thing asteroseismology has not provided us with is the structure of and/or the depth of the surface convection zone. This would help us test the "convective driving" mechanism introduced by Brickhill (1991) and elaborated on by Goldreich \& Wu (1999a,b). Our observations point out the need for further refinements of the parametric instability mechanism described by Wu \& Goldreich (2001) to better cover the observed mode behavior. The observational data set is quite rich, and coupled with more detailed theories, offers the promise of being able to unravel the mysteries of amplitude variation seen in the DBV and DAV white dwarfs. This in turn, may offer us the insights needed to ascertain why only some of the predicted modes are seen at any one time.

Acknowledgements. MAW, AKJ, AEC, and MLB acknowledge support by the National Science Foundation through the Research Experiences for Undergraduates Summer Site Program to Florida Tech.

\section{References}

Althaus, L. G., \& Benvenuto, O. G. 1997, MNRAS, 288, L35

Beauchamp, A., Wesemael, F., Bergeron, P., et al. 1999, ApJ, 516, 887 Bradley, P. A. 2002, in Radial and Nonradial Pulsations as Probes of Stellar Physics, ed. C. Aerts, T. R. Bedding, \& J. Christensen-Dalsgaard, ASP Conf. Ser., 259, 382

Bradley, P. A., \& Winget, D. E. 1994, ApJ, 430, 850

Brassard, P., \& Fontaine, G. 2002, in White Dwarfs, ed. D. Martino, R. Silvotti, J.-E. Solheim, \& R. Kalytis (Dordretch: Kluwer), in press Breger, M., et al. 1993, A\&A, 271, 482

Brickhill, A. J. 1992, MNRAS, 259, 529

Brickhill, A. J. 1991, MNRAS, 251, 673

Brickhill, A. J. 1990, MNRAS, 246, 510

Buchler, J. R., Goupil, M.-J., \& Hansen, C. J. 1997, A\&A, 321, 159

Canuto, V. M., Goldman, I., \& Mazzitelli, I. 1996, ApJ, 473, 550

Clemens, J. C., van Kerkwijk, M. H., \& Wu, Y. 2000, MNRAS, 314, 220

Dehner, B. T., \& Kawaler, S. D. 1995, ApJ, 445, L141

Fontaine, G., \& Brassard, P. 2002, ApJ, 581, L33

Gautschy, A., \& Althaus, L. G. 2002, A\&A, 382, 141

Goldreich, P., \& Wu, Y. 1999a, ApJ, 511, 904

Goldreich, P., \& Wu, Y. 1999b, ApJ, 523, 805 
Ising, J., \& Koester, D. 2001, A\&A, 374, 116

Kawaler, S. D., Sekii, T., \& Gough, D. 1999, ApJ, 516, 349

Kepler, S. O. 1984, ApJ, 286, 314

Kepler, S. O. 1993, Baltic Astron., 2, 515

Kleinman, S. J., Nather, R. E., \& Phillips, T. 1996, PASP, 108, 356

Kleinman, S. J., Nather, R. E., Winget, D. E., et al. 1998, ApJ, 495, 424

Kotak, R., van Kerkwijk, M. H., Clemens, J. C., \& Koester, D. 2003, A\&A, 397, 1043

Kuschnig, R., Weiss, W. W., Gruber, R., Bely, P. Y., \& Jenkner, H. 1997, A\&A, 328, 544

Metcalfe, T. S., \& Charbonneau, P. 2003, J. Comp. Phys., in press

Metcalfe, T. S., Winget, D. E., \& Charbonneau, P. 2001, ApJ, 557, 1021

Metcalfe, T. S., Salaris, M., \& Winget, D. E. 2002, ApJ, 573, 803

Montgomery, M. H., Metcalfe, T. S., \& Winget, D. E. 2001, ApJ, 548, L53

Nather, R. E., Winget, D. E., Clemens, J. C., Hansen, C. J., \& Hine, B. P. 1990, ApJ, 361, 309

Provencal, J. L., Shipman, H. L., Thejll, P., \& Vennes, S. 2000, ApJ, 542,1041
Robinson, E. L., Kepler, S. O., \& Nather, R. E. 1982, ApJ, 259, 219

Scargle, J. D. 1982, ApJ, 263, 835

Schwarzenberg-Czerny, A. 1991, MNRAS, 253, 198

Schwarzenberg-Czerny, A. 1999, ApJ, 516, 315

Shipman, H. L., Provencal, J., Riddle, R., \& Vuckovic, M. 2002, AAS, 200, 7206

Standish, E. M. 1998, A\&A, 336, 381

Thompson, S. E., Clemens, J. C., van Kerkwijk, M. H., \& Koester, D. 2003, ApJ, submitted

van Kerkwijk, M. H., Clemens, J. C., \& Wu, Y. 2000, MNRAS, 314, 209

Vuille, F., et al. 2000, MNRAS, 314, 689

Winget, D. E., Nather, R. E., Clemens, J. C., et al. 1994, ApJ, 430, 839

Winget, D. E., Robinson, E. L., Nather, R. E., Kepler, S. O., \& O'Donoghue, D. 1985, ApJ, 292, 606

Wolff, B., Koester, D., Montgomery, M. H., \& Winget, D. E. 2002, A\&A, 388, 320

Wu, Y. 2001, MNRAS, 323, 248

Wu, Y., \& Goldreich, P. 2001, ApJ, 546, 469 